BULLETIN (New Series) OF THE

AMERICAN MATHEMATICAL SOCIETY

Volume 41, Number 4, Pages 409-438

S 0273-0979(04)01032-8

Article electronically published on June 25, 2004

\title{
A SURVEY OF ENTROPY METHODS FOR PARTIAL DIFFERENTIAL EQUATIONS
}

\author{
LAWRENCE C. EVANS
}

He began then, bewilderingly, to talk about something called entropy ... She did gather that there were two distinct kinds of this entropy. One having to do with heat engines, the other with communication... "Entropy is a figure of speech then"... "a metaphor".

-T. Pynchon, The Crying of Lot 49

\section{INTRODUCTION}

These notes provide for PDE theory a survey of various "entropy methods", by which I mean quantitative and qualitative techniques for understanding irreversibility and dissipation phenomena.

Motivation, ODE examples. I will start slowly, and so introduce the main issues with two simple ODE:

$$
\dot{\mathbf{x}}=-D \Phi(\mathbf{x}) \quad(t>0)
$$

and

$$
\dot{\mathbf{x}}=J D \Phi(\mathbf{x}) \quad(t>0) .
$$

Here $\Phi: \mathbb{R}^{n} \rightarrow \mathbb{R}$ is a given nonnegative potential function and $D \Phi$ denotes the gradient of $\Phi, D \Phi=\left(\Phi_{x_{1}}, \ldots, \Phi_{x_{n}}\right)$. In (2),$J$ denotes some linear operator on $\mathbb{R}^{n}$ satisfying

$$
(J x) \cdot x=0 \quad\left(x \in \mathbb{R}^{n}\right)
$$

think of $J$ as a rotation through a right angle. Hamiltonian systems in particular have the form (2).

We are interested in computing for both dynamics (1) and (2) how $\Phi(\mathbf{x}(t))$ evolves in time. We may calculate for (1)

$$
\frac{d}{d t} \Phi(\mathbf{x})=D \Phi(\mathbf{x}) \cdot \dot{\mathbf{x}}=-|D \Phi(\mathbf{x})|^{2}
$$

Received by the editors April 1, 2004.

2000 Mathematics Subject Classification. Primary 35-02, 35F20, 35K10, 74A15.

LCE was supported in part by NSF Grant DMS-0070480 and by the Miller Institute for Basic Research in Science, UC Berkeley.

(C)2004 American Mathematical Society Reverts to public domain 28 years from publication 
and for (2),

$$
\frac{d}{d t} \Phi(\mathbf{x})=D \Phi(\mathbf{x}) \cdot \dot{\mathbf{x}}=D \Phi(\mathbf{x}) \cdot(J D \Phi(\mathbf{x}))=0 .
$$

So for the evolution (2) the dynamics remain on the level surface $\{\Phi=\Phi(\mathbf{x}(0))\}$; whereas for (11), the mapping $t \mapsto \Phi(\mathbf{x}(t))$ is nonincreasing. For both cases, we therefore have the simple bound

$$
\max _{0 \leq t<\infty} \Phi(\mathbf{x}(t))=\Phi(\mathbf{x}(0)) .
$$

But for problem (1) we have more, since integrating (3) provides us with the additional estimate

$$
\int_{0}^{\infty}|D \Phi(\mathbf{x}(t))|^{2} d t \leq \Phi(\mathbf{x}(0)) .
$$

We interpret the term on the left as recording the total "dissipation" or "irreversibility" of the ODE (11) on the time interval $[0, \infty)$. No similar bound is available for the "conservative" dynamics (2).

So here is a sort-of paradox. Geometrically, we may regard the evolution (2) as simpler than (1), since the latter moves somehow within the full region $\{\Phi \leq$ $\Phi(\mathbf{x}(0))\}$, and not just on the shell $\{\Phi=\Phi(\mathbf{x}(0))\}$. But the dynamics (11) are far better analytically, since the dissipation estimate (5) holds.

PDE examples. This advantage is more clearly seen in a few (much harder) PDE, which are in a sense generalizations of (11).

Navier-Stokes equations. Let $\mathbf{u}=\left(u^{1}, u^{2}, u^{3}\right)$ denote the velocity field and $p$ the pressure in a three-dimensional flow of an incompressible, viscous fluid. These read

$$
\left\{\begin{aligned}
u_{t}^{i}+u^{j} u_{x_{j}}^{i} & =\nu \Delta u^{i}-p_{x_{i}} \quad(i=1,2,3) \\
u_{x_{i}}^{i} & =0 .
\end{aligned}\right.
$$

(In this and subsequent formulas, repeated indices are to be summed.) The constant $\nu>0$ is the inverse of the Reynolds number. Somewhat as in (3), we can calculate

$$
\frac{d}{d t}\left(\int_{\mathbb{R}^{3}} \frac{|\mathbf{u}|^{2}}{2} d x\right)=-\nu \int_{\mathbb{R}^{3}}|D \mathbf{u}|^{2} d x,
$$

the term within the parentheses denoting the kinetic energy. The integrated form of this calculation provides the bound

$$
\int_{0}^{\infty} \int_{\mathbb{R}^{3}}|D \mathbf{u}|^{2} d x d t \leq \frac{1}{2 \nu} \int_{\mathbb{R}^{3}}\left|\mathbf{u}_{0}\right|^{2} d x,
$$

and the expression on the left is very useful, since it controls the gradient $D \mathbf{u}$ of the velocity field. In the right hands, those of Scheffer $[$, Caffarelli-KohnNirenberg [C-K-N], Lin [Li] and others, this is the key to extremely deep and subtle partial regularity assertions for appropriate weak solutions of the Navier-Stokes equations (6). The Euler equations for inviscid, incompressible flow, had by setting $\nu=0$ above, are much harder analytically, since the dissipation estimate (7) is not available.

Mean curvature flow. A geometric problem illustrating the same heuristics is the flow of hypersurfaces by mean curvature. Consider a family of smooth surfaces 
$\left\{\Gamma_{t}\right\}_{t \geq 0}$ evolving in $\mathbb{R}^{n}$ according to the law of motion that

$$
\mathbf{V}=\mathbf{H}
$$

where $\mathbf{V}$ denotes the normal velocity to the surfaces and $\mathbf{H}$ is the mean curvature vector. Then

$$
\frac{d}{d t}\left(\mathcal{H}^{n-1}\left(\Gamma_{t}\right)\right)=-\int_{\Gamma_{t}} \mathbf{V} \cdot \mathbf{H} d \mathcal{H}^{n-1}=-\int_{\Gamma_{t}} H^{2} d \mathcal{H}^{n-1}
$$

and $H$ denotes $n-1$ times the mean curvature; $\mathcal{H}^{n-1}$ means $n-1$ dimensional surface measure. The analogue of (5) is then

$$
\int_{0}^{\infty} \int_{\Gamma_{t}} H^{2} d \mathcal{H}^{n-1} d t \leq \mathcal{H}^{n-1}\left(\Gamma_{0}\right)
$$

This geometric "dissipation" estimate, providing an $L^{2}$ bound on the mean curvature of the evolving surface, lies at the heart of Brakke's magnificent work $[B]$ on the structure and partial regularity of generalized solutions to mean curvature motion flow. See Ecker's new book $[\mathrm{Ec}]$ for more.

First-order PDE. In fact, the dissipation effects I have been discussing are so strong that "even when they are not there" they still control the solutions of certain nonlinear first-order PDE. What I mean by this odd pronouncement is that the limits of solutions to approximating equations with small dissipation are usually profoundly affected as the dissipation rate goes to zero. This means in practice that for "weak solutions" of the first-order PDE so constructed certain types of singularities can be ruled out as nonadmissible. The examples discussed below in sections $6-10$ will illustrate more precisely what I mean.

Overview. My rationale for these notes is that the foregoing calculations suggest the possibility of some kind of a unified analytic approach to various nonlinear PDE displaying irreversibility. I have made a really quite idiosyncratic and eclectic selection of the illustrative topics below. I will argue that the procedures above can, heuristically at least, be profitably understood as "entropy" methods, and in particular that dissipation inequalities of the type (5), (7), (9), etc., are variants of the Second Law of Thermodynamics and in particular the classical Clausius inequality. I will therefore regard "entropy" sometimes as a physical quantity, but more often as a metaphor.

This paper is a much belated revision of notes I provided for the Colloquium Lectures I gave at the Joint Mathematics Meetings in San Diego, January 2002. Readers interested in details missing here can download from my website at the UC Berkeley Mathematics Department lecture notes for a semester course I taught on some of this material.

\section{REVIEW OF CLASSICAL THERMODYNAMiCS}

(Callen [C], Wightman [W], Dittman-Zemansky [D-Ze]).

This section provides a rushed overview of thermodynamics, primarily the axiomatic development. I intend both to sketch in background for subsequent PDE discussions and also to advertise some fascinating mathematical issues. 


\subsection{A model for a thermal system in equilibrium.}

Notation: $\left(X_{0}, X_{1}, \ldots, X_{m}\right) \in \Sigma \subset \mathbb{R}^{m+1}, E=X_{0} . \Sigma$ is the state space and $E$ is the internal energy.

Assume we are given $S: \Sigma \rightarrow \mathbb{R}$ such that

$$
S \text { is concave, } \frac{\partial S}{\partial E}>0 \text {, and } S \text { is positively homogeneous of degree } 1 .
$$

We call $S$ the entropy of our system: $S=S\left(E, X_{1}, \ldots, X_{m}\right)$. Now solve for $E=$ $E\left(S, X_{1}, \ldots, X_{m}\right)$ and define

$$
\left\{\begin{aligned}
T & =\frac{\partial E}{\partial S}=\text { temperature } \\
P_{k} & =-\frac{\partial E}{\partial X_{k}}=k^{t h} \text { generalized force (or pressure) } .
\end{aligned}\right.
$$

Then

$$
\frac{\partial S}{\partial E}=\frac{1}{T}, \frac{\partial S}{\partial X_{k}}=\frac{P_{k}}{T} \quad(k=1, \ldots, m) .
$$

\subsection{Thermodynamic potentials.}

A. Legendre transform. Assume that $H: \mathbb{R}^{n} \rightarrow(-\infty,+\infty]$ is a convex, lower semicontinuous function, which is proper (i.e. $\not \equiv+\infty$ ). The Legendre transform of $L$ is

$$
L(q):=\sup _{p \in \mathbb{R}^{n}}(p \cdot q-H(p)) .
$$

We write $L=H^{*}$. Then $L$ is likewise convex, lower semicontinuous and proper, and $L^{*}=H$. If $H$ is also $C^{2}$ and strictly convex, then $L(q)=p \cdot q-H(p)$, for the unique point $p=p(q)$ solving $q=D H(p)$.

B. Definitions. We hereafter write $E=E\left(S, V, X_{2}, \ldots, X_{m}\right)=E(S, V)$.

The Helmholtz free energy $F$ is

$$
F(T, V)=\inf _{S}(E(S, V)-T S) .
$$

The enthalpy $H$ is

$$
H(S, P)=\inf _{V}(E(S, V)+P V) .
$$

The Gibbs potential is

$$
G(T, P)=\inf _{S, V}(E(S, V)+P V-S T) .
$$

These definitions are variants of the standard Legendre transform for convex functions. We call $E, F, G, H$ thermodynamic potentials.

\section{Formulas for partial derivatives:}

$$
\begin{aligned}
& \frac{\partial E}{\partial S}=T, \quad \frac{\partial E}{\partial V}=-P, \\
& \frac{\partial F}{\partial T}=-S, \frac{\partial F}{\partial V}=-P, \\
& \frac{\partial G}{\partial T}=-S, \frac{\partial G}{\partial P}=V \\
& \frac{\partial H}{\partial S}=T, \quad \frac{\partial H}{\partial P}=V .
\end{aligned}
$$




\section{Capacities.}

$$
\begin{aligned}
& C_{P}=T\left(\frac{\partial S}{\partial T}\right)_{P}=\text { heat capacity at constant pressure } \\
& C_{V}=T\left(\frac{\partial S}{\partial T}\right)_{V}=\text { heat capacity at constant volume } \\
& \Lambda_{V}=T\left(\frac{\partial S}{\partial V}\right)_{T}=\text { latent heat with respect to volume }
\end{aligned}
$$

2.3. Thermodynamic processes. (Owen $\mathrm{O}$, Bharatha-Truesdell $\mathrm{B}-\mathrm{T}]$ )

The next sections are to publicize some mathematical models within which we can formulate forms of the First and Second Laws of Thermodynamics and deduce as consequences the existence of the energy $E$ and entropy $S$.

A. A model for a homogeneous fluid body without dissipation. We are given functions $P=P(T, V), \Lambda_{V}=\Lambda_{V}(T, V)$ and $C_{V}=C_{V}(T, V)$, satisfying

$$
\frac{\partial P}{\partial V}<0, \quad \Lambda_{V} \neq 0, \quad C_{V}>0
$$

Let $\Gamma=\{(T(t), V(t)) \mid a \leq t \leq b\}$ be a path connecting the state $A=(T(a), V(a))$ to the state $B=(T(b), V(b))$. Call $\Gamma$ a cycle if $A=B$. Let us also write

$$
\mathbb{W}(\Gamma):=\int_{\Gamma} P d V=\text { work done by the fluid }
$$

and

$$
\mathbb{Q}(\Gamma):=\int_{\Gamma} C_{V} d T+\Lambda_{V} d V=\text { heat gained by the fluid. }
$$

We hypothesize:

First Law of Thermodynamics: For every cycle $\Gamma$, we have $\mathbb{W}(\Gamma)=\mathbb{Q}(\Gamma)$.

As is shown in the cited references, from this axiom follows the existence of an internal energy function:

Theorem 1. There exists a function $E=E(T, V)$ such that

$$
\frac{\partial E}{\partial V}=\Lambda_{V}-P, \frac{\partial E}{\partial T}=C_{V} .
$$

We next define a Carnot cycle $\Gamma$ to be a cycle as drawn in Figure 1, consisting of two adiabatic paths $\Gamma_{b}, \Gamma_{d}$ (along which there is no heating) and two isothermal paths $\Gamma_{a}, \Gamma_{c}$.

Define $\mathbb{Q}^{+}(\Gamma)$ to be the heat gained along the isothermal path $\Gamma_{a}$, at the higher temperature $T_{2}$.

Second Law of Thermodynamics: For each Carnot heat engine $\Gamma$ as above, we have

$$
0<\mathbb{W}(\Gamma)=\left(1-\frac{T_{1}}{T_{2}}\right) \mathbb{Q}^{+}(\Gamma)
$$

The references explain how formula (13) in fact "follows physically" from this more familiar statement that "there is no thermodynamic process the sole result of which is that heat is transmitted from a body to a hotter body". A consequence is the existence of an entropy function: 


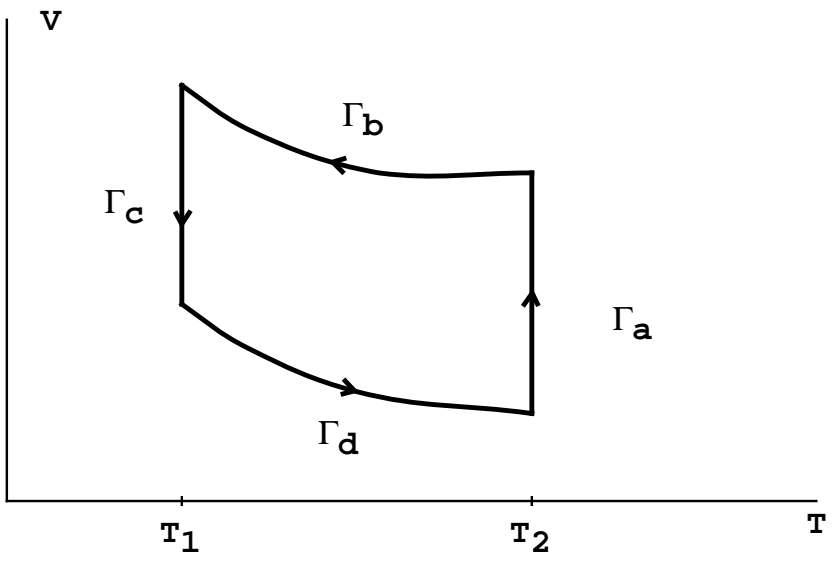

FIGURE 1.

Theorem 2. There exists a function $S: \Sigma \rightarrow \mathbb{R}$ such that

$$
\frac{\partial S}{\partial V}=\frac{\Lambda_{V}}{T}, \frac{\partial S}{\partial T}=\frac{C_{V}}{T} .
$$

Please see the cited references Owen [O] and Bharatha-Truesdell [B-T] for full discussions of these and related mathematical models.

B. A model with dissipation. The previous model is elegant mathematically, but does not admit the notion of "irreversibility". Following Serrin [S1], let us now redefine

$$
\mathbb{W}(\Gamma)=\int_{a}^{b} P(T, V) \dot{V}+R_{1}(T, V, \dot{T}, \dot{V}) d t
$$

and

$$
\mathbb{Q}(\Gamma)=\int_{a}^{b} C_{V}(T, V) \dot{T}+\Lambda_{V}(T, V) \dot{V}+R_{2}(T, V, \dot{T}, \dot{V}) d t .
$$

Here $R_{1}, R_{2}$ are new dissipation terms, which are assumed to be quadratic in $\dot{T}, \dot{V}$. The First and Second Laws imply the existence of $E$ and $S$, as before, since our new model approximates a dissipationless model if we reparameterize on slower and slower time scales. Thus also $R_{1} \equiv R_{2}$.

Finally, we assume $R_{1}(T, V, \dot{T}, \dot{V})=R_{2}(T, V, \dot{T}, \dot{V}) \leq 0$. Then for any cyclic process $\Gamma$, we can define

$$
\mathcal{Q}(T, V, \dot{T}, \dot{V}):=C_{V}(T, V) \dot{T}+\Lambda_{V}(T, V) \dot{V}+R_{2}(T, V, \dot{T}, \dot{V})
$$

and compute

$$
\int_{0}^{b} \frac{\mathcal{Q}(T, V, \dot{T}, \dot{V})}{T} d t=\int_{a}^{b} \frac{d}{d t} S(T, V) d t+\int_{a}^{b} \frac{R_{2}(T, V, \dot{T}, \dot{V})}{T} d t \leq 0 .
$$

We introduce new notation and rewrite:

$$
\int_{\Gamma} \frac{d Q}{T} \leq 0 \quad(\Gamma \text { a cyclic process }) .
$$


This is a form of Clausius' inequality. If we take a process connecting a state $A$ to a state $B$, we similarly deduce

$$
\int_{\Gamma} \frac{d Q}{T} \leq S(B)-S(A) \quad(\Gamma \text { a process from A to B }) .
$$

One of our goals in these notes is identifying for various PDE dissipation inequalities that can be seen as variants of Clausius' inequality.

See also Day-Šilhavý [D-S], Serrin [S1], S2], Coleman-Owen-Serrin [C-O-S] and Feinberg-Lavine $[\mathrm{F}-\mathrm{L}$ ] for general derivations based upon different mathematical interpretations of the Second Law. A novel approach has been introduced by Lieb and Yngvason [L-Y].

The December 1999 theme issue of the American Journal of Physics on thermal and statistical physics AJP is filled with interesting articles, accessible to mathematicians.

\section{Continuum thermodynamics}

(Coleman-Noll [C-N], Ericksen [Er], Gurtin-Williams G-W]).

The foregoing models do not admit any spatial dependence in the relevant variables. Since the intention is later to discuss dissipation effects in partial differential equations, we must introduce dependence of physical variables upon position $x$ and time $t$. Mostly following Coleman-Noll $[\mathrm{C}-\mathrm{N}]$, we hypothesize a local form of the Clausius' inequality, and this combined with basic physical conservation laws and constitutive rules lets us deduce certain local forms of the thermodynamic principles mentioned in $\S 2$. As before, to save space we leave out all the interesting details of the derivations.

\subsection{Physical principles.}

- Physical quantities:

$$
\begin{array}{ll}
e(x, t)=\text { internal energy/unit mass, } & \mathbf{v}(x, t)=\text { velocity }, \\
\mathbf{b}(x, t)=\text { body force/unit mass } & \rho(x, t)=\text { mass density }, \\
\mathbf{q}(x, t)=\text { heat flux vector, } & s(x, t)=\text { entropy } / \text { unit mass } \\
r(x, t)=\text { heat supply/unit mass, } & \theta(x, t)=\text { local temperature, }
\end{array}
$$$$
\mathbf{T}(x, t)=\text { stress tensor. }
$$

\section{- Basic physical laws:}

$$
\begin{gathered}
\frac{\partial \rho}{\partial t}+\operatorname{div}(\rho \mathbf{v})=0 \quad \text { (conservation of mass). } \\
\rho \frac{D \mathbf{v}}{D t}=\rho \mathbf{b}+\operatorname{div} \mathbf{T} \quad \text { (balance of momentum). } \\
\rho \frac{D e}{D t}=\rho r-\operatorname{div} \mathbf{q}+\mathbf{T}: D \mathbf{v} \quad \text { (energy balance). } \\
\rho \frac{D s}{D t} \geq \frac{r \rho}{\theta}-\operatorname{div}\left(\frac{\mathbf{q}}{\theta}\right) \quad \text { (Clausius-Duhem inequality). }
\end{gathered}
$$

In these formulas, $D$ is the gradient $\left(\frac{\partial}{\partial x_{1}}, \ldots, \frac{\partial}{\partial x_{n}}\right)$, and $\frac{D f}{D t}=\frac{\partial f}{\partial t}+\mathbf{v} \cdot D f$ is the material derivative. The local production of entropy per unit mass is

$$
\gamma:=\frac{D s}{D t}-\frac{r}{\theta}+\frac{1}{\rho} \operatorname{div}\left(\frac{\mathbf{q}}{\theta}\right) \geq 0 .
$$


3.2. Constitutive relations. A particular material is defined by adding to the foregoing additional constitutive relations.

- Example: fluids (Coleman-Noll $[\mathrm{C}-\mathrm{N}]$ ). We introduce the specific volume $v=\frac{1}{\rho}$ and call our body a perfect fluid with heat conduction if there exist four functions $\hat{e}, \hat{\theta}, \hat{T}, \hat{\mathbf{q}}$ such that

$$
\left\{\begin{array}{l}
e=\hat{e}(s, v), \quad \theta=\hat{\theta}(s, v) \\
\mathbf{T}=\hat{\mathbf{T}}(s, v), \quad \mathbf{q}=\hat{\mathbf{q}}(s, v, D \theta) .
\end{array}\right.
$$

After various substitutions using the physical laws above, we can derive the inequality

$$
0 \leq \rho\left(\hat{\theta}-\frac{\partial \hat{e}}{\partial s}\right) \frac{D s}{D t}+\left(\hat{T}-\frac{\partial \hat{e}}{\partial v} I\right): D \mathbf{v}-\frac{1}{\theta} \hat{\mathbf{q}} \cdot D \theta .
$$

This inequality must hold for all admissible thermodynamic processes. Taking various choices and dropping the circumflex in our notation, we can conclude

$$
\begin{gathered}
\frac{\partial e}{\partial s}=\theta \quad \text { (temperature formula), } \\
\mathbf{T}=-p I, \quad \text { for } \frac{\partial e}{\partial v}=-p \quad \text { (pressure formula), } \\
\mathbf{q}(s, v, p) \cdot p \leq 0 \quad \text { (heat conduction inequality). }
\end{gathered}
$$

See Coleman-Noll [C-N] for the specifics of all this. Also, compare (16) and (17) with (12).

- Example: heat conduction in a rigid body (Gurtin Gu $)$. Now assume $\mathbf{v} \equiv 0, \mathbf{b} \equiv 0, \rho \equiv 1$. We introduce the constitutive relations

$$
e=\hat{e}(\theta, D \theta), \quad s=\hat{s}(\theta, D \theta), \quad \mathbf{q}=\hat{\mathbf{q}}(\theta, D \theta) .
$$

It turns out then that $e=e(\theta), s=s(\theta)$. We derive from this the general heat conduction equation

$$
c_{v}(\theta) \frac{\partial \theta}{\partial t}+\operatorname{div}(\mathbf{q}(\theta, D \theta))=r .
$$

The heat capacity/unit mass is $c_{v}(\theta):=e^{\prime}(\theta)$, and if $r \equiv 0$, local entropy production is

$$
\gamma=\frac{-\mathbf{q}(\theta, D \theta) \cdot D \theta}{\theta^{2}}
$$

Our first model in $\S 2.3$ corresponds to dissipationless work, and this model entails workless dissipation.

Remark. The heat conduction inequality (18) holds here as well. It is however disturbing that the Clausius-Duhem inequality (15) apparently does not imply the stronger monotonicity condition

$$
\left(\mathbf{q}\left(\theta, p_{1}\right)-\mathbf{q}\left(\theta, p_{2}\right)\right) \cdot\left(p_{1}-p_{2}\right) \leq 0
$$

for all $p_{1}, p_{2}$. Condition (20) would say that the PDE (19) is parabolic and thus well-posed forward in time.

\section{The heAT EQUATION}

Turning at last to PDE theory proper, we first examine the implications of the foregoing for the linear heat equation. 
4.1. Entropy increase. A special case of (19) is the nonhomogeneous heat equation

$$
u_{t}-\Delta u=f \quad \text { in } U \times[0, \infty)
$$

where $\Delta u:=\sum_{i=1}^{n} u_{x_{i} x_{i}}$ is the Laplacian of $u, U$ is a bounded, smooth region, and $\frac{\partial u}{\partial \nu}=0$ on $\partial U$. We think of (21) as a heat conduction PDE, with

$$
\left\{\begin{array}{l}
u=\text { temperature, } u>0 \\
\mathbf{q}=-D u=\text { heat flux, } \\
f=\text { heat supply/unit mass, } f \geq 0,
\end{array}\right.
$$

and the heat capacity is $c_{v} \equiv 1$. Also, up to additive constants, we have

$$
\left\{\begin{aligned}
u & =\text { internal energy/unit mass } \\
\log u & =\text { entropy/unit mass. } \\
\frac{|D u|^{2}}{u^{2}} & =\gamma=\text { local production of entropy. }
\end{aligned}\right.
$$

Define

$$
\begin{gathered}
S(t):=\int_{U} \log u(x, t) d x=\text { entropy at time } t, \\
F(t):=\int_{U} \frac{f(x, t)}{u(x, t)} d x=\text { entropy supply, } \\
G(t):=\int_{U} \gamma(x, t) d x=\text { rate of internal entropy generation. }
\end{gathered}
$$

A simple calculation establishes

Theorem 3. Assume u solves (21). Then

$$
\frac{d S}{d t}=F+G \geq 0 .
$$

This is a statement of entropy increase, the sort of thermodynamic-like assertion we are looking for. But this is really not so impressive, since in fact

$$
t \mapsto \int_{U} \Phi(u(x, t)) d x
$$

is nonincreasing if $\Phi$ is any smooth function satisfying $\Phi^{\prime} \leq 0, \Phi^{\prime \prime} \geq 0$ :

$$
\frac{d}{d t} \int_{U} \Phi(u) d x=\int_{U} \Phi^{\prime}(u) u_{t} d x=\int_{U} \Phi^{\prime}(u)(\Delta u+f) d x \leq-\int_{U} \Phi^{\prime \prime}(u)|D u|^{2} d x \leq 0 .
$$

4.2. A differential form of Harnack's inequality. (Li-Yau L-Ya]) Is there really anything special about the particular choice of $\Phi(u)=-\log u$ ? Let us again consider positive solutions $u$ of the heat equation, for $f \equiv 0$. We further assume $U$ is convex.

Theorem 4. (i) We have

$$
\frac{u_{t}}{u}+\frac{n}{2 t} \geq \frac{|D u|^{2}}{u^{2}} .
$$

(ii) Furthermore, for each $x_{1}, x_{2} \in \bar{U}$ and $0<t_{1}<t_{2}$, this estimate holds:

$$
u\left(x_{1}, t_{1}\right) \leq\left(\frac{t_{2}}{t_{1}}\right)^{n / 2} e^{\frac{\left|x_{2}-x_{1}\right|^{2}}{4\left(t_{2}-t_{1}\right)}} u\left(x_{2}, t_{2}\right) .
$$


Note that we can rewrite (23) as the pointwise thermodynamic bound

$$
s_{t}+\frac{n}{2 t} \geq \gamma \text {. }
$$

The estimate (24) is a form of Harnack's inequality for the heat equation.

Idea of proof. 1. Write $v=\log u$ so that the heat equation transforms into

$$
v_{t}-\Delta v=|D v|^{2} .
$$

Set $w=\Delta v$ and $\tilde{w}:=t w+\frac{n}{2}$. Then an estimate exploiting the good term on the right hand side of (25) shows that

$$
\tilde{w}_{t}-\Delta \tilde{w}-2 D v \cdot D \tilde{w} \geq-\frac{1}{t} \tilde{w} .
$$

It turns out furthermore that $\frac{\partial \tilde{w}}{\partial \nu} \geq 0$ on $\partial U \times[0, \infty)$. The maximum principle therefore implies

$$
\tilde{w}=t w+\frac{n}{2} \geq 0 .
$$

But $w=\Delta v=v_{t}-|D v|^{2}=\frac{u_{t}}{u}-\frac{|D u|^{2}}{u^{2}}$, and estimate (23) follows.

2. We may further compute

$$
\begin{aligned}
v\left(x_{2}, t_{2}\right)-v\left(x_{1}, t_{1}\right) & =\int_{0}^{1} D v \cdot\left(x_{2}-x_{1}\right)+v_{t}\left(t_{2}-t_{1}\right) d s \\
& \geq \int_{0}^{1}-|D v|\left|x_{2}-x_{1}\right|+\left(|D v|^{2}-\frac{n}{2\left(s t_{2}+(1-s) t_{1}\right)}\right)\left(t_{2}-t_{1}\right) d s \\
& \geq-\frac{n}{2} \log \left(\frac{t_{2}}{t_{1}}\right)-\frac{\left|x_{2}-x_{1}\right|^{2}}{4\left(t_{2}-t_{1}\right)} .
\end{aligned}
$$

Exponentiate.

4.3. Clausius' inequality for the heat equation. Day's very interesting book D] is filled with assertions for the heat equation that have close analogies in thermodynamics. We present next a sample such calculation.

We hereafter assume $u>0$ is a smooth solution of the heat equation, with

$$
u(\cdot, t)=\tau(t) \quad \text { on } \partial U,
$$

where $\tau$ is a given nonnegative function. Let us assume that $\tau$ is T-periodic: $\tau(t+T)=\tau(t)$ for all $t \geq 0$, and call a $T$-periodic solution $u$ a cycle.

Theorem 5. Corresponding to each smooth T-periodic function $\tau$ as above, there exists a unique cycle $u$.

Idea of proof. Given a smooth function $g$, we denote by $u$ the unique smooth solution of

$$
\left\{\begin{array}{rll}
u_{t}-\Delta u=0 & \text { in } U \times(0, T] \\
u=\tau & \text { on } \partial U \times[0, T] \\
u=g & \text { on } U \times\{t=0\} .
\end{array}\right.
$$

The mapping $g \mapsto u(\cdot, T)$ extends to a strict contraction on $L^{2}$ and so has a unique fixed point.

Let $u$ be the unique cycle corresponding to $\tau$ and define

$$
Q(t):=\int_{\partial U} \frac{\partial u}{\partial \nu} d S,
$$

the total heat flux into $U$ from its exterior, at time $t \geq 0$. 
Theorem 6. We have

$$
\int_{0}^{T} \frac{Q}{\tau} d t \leq 0
$$

with strict inequality unless $\tau$ is constant.

This of course is a version of Clausius' inequality (14).

Idea of proof. Write $v=\log u$ so that as before $v_{t}-\Delta v=|D v|^{2}=\gamma \geq 0$. Then

$$
\frac{d}{d t}\left(\int_{U} v d x\right)=\int_{\partial U} \frac{\partial v}{\partial \nu} d S+\int_{U} \gamma d x \geq \int_{\partial U} \frac{1}{u} \frac{\partial u}{\partial \nu} d S=\frac{Q(t)}{\tau(t)}
$$

since $u(\cdot, t)=\tau(t)$ on $\partial U$. Since $v$ is periodic in time, we deduce (26) upon integrating.

\section{Some Physical PARTIAL DiffERENTIAL EQUations}

For later reference, we recount the structure of several important nonlinear PDE in continuum physics.

5.1. Compressible Euler equations. These are the PDE for inviscid, isentropic fluid flow:

$$
\left\{\begin{aligned}
\frac{D \rho}{D t}+\rho \operatorname{div} \mathbf{v} & =0 \\
\rho \frac{D \mathbf{v}}{D t} & =-D p .
\end{aligned}\right.
$$

We can rewrite these in conservation form

$$
\left\{\begin{aligned}
\rho_{t}+\operatorname{div}(\rho \mathbf{v}) & =0 \\
(\rho \mathbf{v})_{t}+\operatorname{div}(\rho \mathbf{v} \otimes \mathbf{v}+p I) & =0
\end{aligned}\right.
$$

where $\mathbf{v} \otimes \mathbf{v}=\left(\left(v^{i} v^{j}\right)\right)$ and $p=p(\rho)$.

5.2. Boltzmann's equation. Boltzmann's equation is the integro/differential equation

$$
f_{t}+v \cdot D_{x} f=Q(f, f)
$$

for a certain quadratic collision operator $Q$. This term models the rate of collisions which start with velocity pairs $v, v_{*}$ and result in velocity pairs $v^{\prime}, v_{*}^{\prime}$. The unknown is $f=f(x, v, t)$, the density of the number of particles at time $t$ and position $x$, with velocity $v$.

Assume $f>0$ is a smooth solution, and define Boltzmann's $H$-function

$$
H(t):=\int_{\mathbb{R}^{3}} \int_{\mathbb{R}^{3}} f \log f d v d x .
$$

Then

$$
\frac{d H}{d t} \leq 0
$$

A physical argument suggests the interpretation

$$
S=-k H,
$$

where $k$ is Boltzmann's constant. So (28) is another variant of Clausius' inequality. A function $f=f(v)$ is called a Maxwellian if $Q(f, f) \equiv 0$, in which case $f$ has the form: $f(v)=a e^{-b|v-c|^{2}}$ for constants $a, b, c$. The proof of (28) shows that we have $\frac{d}{d t} H(t)<0$ unless $v \mapsto f(x, v, t)$ is a Maxwellian. 


\section{Conservation laws}

6.1. Terminology, integral and entropy solutions. (Lax [Lx1]) A PDE of the form

$$
u_{t}+\operatorname{div} \mathbf{F}(u)=0 \quad \text { in } \mathbb{R}^{n} \times(0, \infty)
$$

is called a conservation law. The unknown is $u$, and we are given the flux function $\mathbf{F}=\left(F^{1}, \ldots, F^{n}\right)$. We will sometimes rewrite (29) into nondivergence form

$$
u_{t}+\mathbf{b}(u) \cdot D u=0,
$$

for $\mathbf{b}=\mathbf{F}^{\prime}$.

We will in particular study the initial value problem

$$
\left\{\begin{aligned}
u_{t}+\operatorname{div} \mathbf{F}(u) & =0 & & \text { in } \mathbb{R}^{n} \times(0, \infty) \\
u & =g & & \text { on } \mathbb{R}^{n} \times\{t=0\},
\end{aligned}\right.
$$

where $g \in L_{\text {loc }}^{1}$ is the initial density.

Definition. We say $u \in L_{\text {loc }}^{1}$ is an integral solution of (31) provided

$$
\int_{0}^{\infty} \int_{\mathbb{R}^{n}} u v_{t}+\mathbf{F}(u) \cdot D v d x d t+\int_{\mathbb{R}^{n}} g v(\cdot, 0) d x=0
$$

for all $v \in C_{c}^{1}$.

Definition. We call $(\Phi, \Psi)$ an entropy/entropy flux pair for the conservation law (29) provided $\Phi: \mathbb{R} \rightarrow \mathbb{R}$ is convex, and $\boldsymbol{\Psi}: \mathbb{R} \rightarrow \mathbb{R}^{n}, \Psi=\left(\Psi^{1}, \ldots, \Psi^{n}\right)$ satisfies

$$
\Psi^{\prime}=\mathbf{b} \Phi^{\prime}
$$

Motivation. Introduce for $\varepsilon>0$ the regularized PDE

$$
u_{t}^{\varepsilon}+\operatorname{div} \mathbf{F}\left(u^{\varepsilon}\right)=\varepsilon \Delta u^{\varepsilon} .
$$

Take a smooth entropy/entropy flux pair $\Phi, \Psi$ and compute:

$$
\begin{aligned}
\Phi\left(u^{\varepsilon}\right)_{t}+\operatorname{div} \boldsymbol{\Psi}\left(u^{\varepsilon}\right) & =\Phi^{\prime}\left(u^{\varepsilon}\right)\left(-\mathbf{b}\left(u^{\varepsilon}\right) \cdot D u^{\varepsilon}+\varepsilon \Delta u^{\varepsilon}\right)+\Psi^{\prime}\left(u^{\varepsilon}\right) \cdot D u^{\varepsilon} \\
& =\varepsilon \Phi^{\prime}\left(u^{\varepsilon}\right) \Delta u^{\varepsilon} \\
& =\varepsilon \operatorname{div}\left(\Phi^{\prime}\left(u^{\varepsilon}\right) D u^{\varepsilon}\right)-\varepsilon \Phi^{\prime \prime}\left(u^{\varepsilon}\right)\left|D u^{\varepsilon}\right|^{2} \\
& \leq \varepsilon \operatorname{div}\left(\Phi^{\prime}\left(u^{\varepsilon}\right) D u^{\varepsilon}\right) .
\end{aligned}
$$

Definition. We say that $u$ is an entropy solution provided

$$
\Phi(u)_{t}+\operatorname{div} \boldsymbol{\Psi}(u) \leq 0
$$

in the distribution sense for each entropy/entropy flux pair $(\Phi, \Psi)$.

6.2. Jump conditions across shocks. Assume that $n=1$ and that some region $V$ is subdivided into regions $V_{l}, V_{r}$ by a curve $C$; see Figure 2 .

Assume that $u$ is smooth in $\bar{V}_{l}, \bar{V}_{r}$ and also satisfies the entropy condition (32). Take $\Phi(z)= \pm z, \Psi(z)= \pm F(z)$, to conclude

$$
u_{t}+F(u)_{x}=0 \quad \text { in } V_{l}, V_{r} .
$$

Next take $v \in C_{c}^{1}, v \geq 0$. Then (32) implies

$$
\iint_{V_{t}} \Phi(u) v_{t}+\Psi(u) v_{x} d x d t+\iint_{V_{r}} \Phi(u) v_{t}+\Psi(u) v_{x} d x d t \geq 0 .
$$




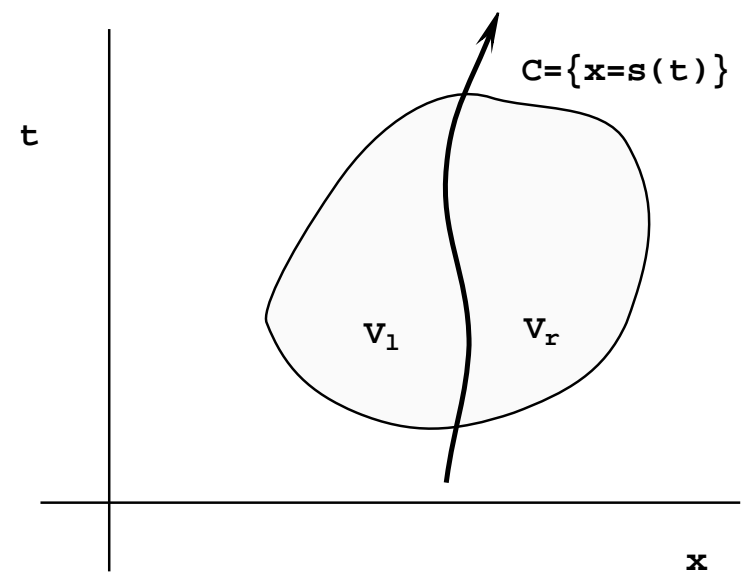

FIGURE 2.

Integrate by parts to deduce

$$
\int_{C} v\left[\left(\Phi\left(u_{l}\right)-\Phi\left(u_{r}\right)\right) \nu^{2}+\left(\Psi\left(u_{l}\right)-\Psi\left(u_{r}\right)\right) \nu^{1}\right] d H^{1} \geq 0
$$

where $\boldsymbol{\nu}=\left(\nu^{1}, \nu^{2}\right)$ is the outer unit normal to $V_{l}$ along $C$. We conclude that

$$
\dot{s}\left(\Phi\left(u_{r}\right)-\Phi\left(u_{l}\right)\right) \geq \Psi\left(u_{r}\right)-\Psi\left(u_{l}\right) \quad \text { along } C .
$$

Taking $\Phi(z)= \pm z, \Psi(z)= \pm F(z)$, we derive the Rankine-Hugoniot jump condition

$$
\dot{s}[u]=[F(u)],
$$

for $[u]:=u_{r}-u_{l},[F(u)]:=F\left(u_{r}\right)-F\left(u_{l}\right)$.

Suppose $u_{l}<u_{r}$. Fix $u_{l}<u<u_{r}$ and define the entropy/entropy flux pair

$$
\left\{\begin{array}{l}
\Phi(z):=(z-u)_{+} \\
\Psi(z):=\int_{u_{l}}^{z} \operatorname{sgn}_{+}(v-u) F^{\prime}(v) d v .
\end{array}\right.
$$

Then

$$
\left\{\begin{array}{l}
\Phi\left(u_{r}\right)-\Phi\left(u_{l}\right)=u_{r}-u \\
\Psi\left(u_{r}\right)-\Psi\left(u_{l}\right)=F\left(u_{r}\right)-F(u) .
\end{array}\right.
$$

Consequently (33) implies

$$
\dot{s}\left(u-u_{r}\right) \leq F(u)-F\left(u_{r}\right) .
$$

Combine (34), (35)):

$$
F(u) \geq\left[\frac{F\left(u_{r}\right)-F\left(u_{l}\right)}{u_{r}-u_{l}}\right]\left(u-u_{r}\right)+F\left(u_{r}\right) \quad\left(u_{l} \leq u \leq u_{r}\right) .
$$

Likewise, if $u_{l}>u_{r}$, then

$$
F(u) \leq\left[\frac{F\left(u_{r}\right)-F\left(u_{l}\right)}{u_{r}-u_{l}}\right]\left(u-u_{r}\right)+F\left(u_{r}\right) \quad\left(u_{r} \leq u \leq u_{l}\right) .
$$

The inequalities (36), (37) are Oleinik's condition E. 
6.3. Systems of conservation laws. A system of conservation laws is written

$$
\mathbf{u}_{t}+\operatorname{div} \mathbf{F}(\mathbf{u})=0 \quad \text { in } \mathbb{R}^{n} \times(0, \infty),
$$

for which the unknown is $\mathbf{u}=\left(u^{1}, \ldots, u^{m}\right)$ and the flux function

$$
\mathbf{F}=\left(\begin{array}{ccc}
F_{1}^{1} & \ldots & F_{n}^{1} \\
\vdots & & \vdots \\
F_{1}^{m} & \ldots & F_{n}^{m}
\end{array}\right)_{m \times n}
$$

is given.

We are interested in properly formulating the initial value problem

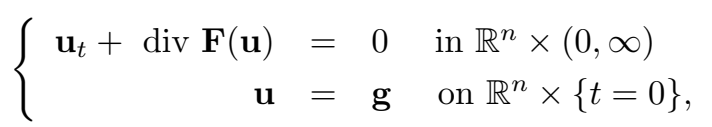

for given $\mathbf{g}$. Let us say $\mathbf{u} \in L_{\mathrm{loc}}^{1}$ is an integral solution of (39) provided

$$
\int_{0}^{\infty} \int_{\mathbb{R}^{n}} \mathbf{u} \cdot \mathbf{v}_{t}+\mathbf{F}(\mathbf{u}): D \mathbf{v} d x d t+\int_{\mathbb{R}^{n}} \mathbf{g} \cdot \mathbf{v}(\cdot, 0) d x=0
$$

for each $\mathbf{v} \in C_{c}^{1}$.

We call $(\Phi, \boldsymbol{\Psi})$ an entropy/entropy flux pair for the conservation law (38) provided $\Phi: \mathbb{R}^{m} \rightarrow \mathbb{R}$ is convex and $\boldsymbol{\Psi}: \mathbb{R}^{m} \rightarrow \mathbb{R}^{n}, \Psi=\left(\Psi^{1}, \ldots, \Psi^{n}\right)$, satisfies

$$
D \Psi=\mathbf{B} D \Phi
$$

for $\mathbf{B}=D \mathbf{F}$.

Unlike for scalar conservation laws, it may be difficult or impossible to find any entropy/entropy flux pairs for a given system.

- Example: compressible Euler equations. We consider now the compressible Euler equations in one space dimension. These have the form $\mathbf{u}_{t}+\mathbf{F}(\mathbf{u})_{x}=0$ for $m=2$ and

$$
\left\{\begin{array}{l}
\mathbf{u}=(\rho, \rho v) \\
\mathbf{F}=\left(z_{2}, z_{2}^{2} / z_{1}+p\left(z_{1}\right)\right) .
\end{array}\right.
$$

We look for entropy/entropy flux pairs, and to simplify subsequent calculations take $\Phi, \Psi$ to be functions of $(\rho, v)$. First, rewrite Euler's equations into nondivergence form:

and compute

$$
\left\{\begin{aligned}
\rho_{t}+\rho_{x} v+\rho v_{x} & =0 \\
v_{t}+v v_{x} & =-\frac{1}{\rho} p_{x}=-p^{\prime} \frac{\rho_{x}}{\rho},
\end{aligned}\right.
$$

$$
\begin{aligned}
\Phi_{t}+\Psi_{x} & =\Phi_{\rho} \rho_{t}+\Phi_{v} v_{t}+\Psi_{\rho} \rho_{x}+\Psi_{v} v_{x} \\
& =\Phi_{\rho}\left(-\rho_{x} v-\rho v_{x}\right)+\Phi_{v}\left(-v v_{x}-p^{\prime} \frac{\rho_{x}}{\rho}\right)+\Psi_{\rho} \rho_{x}+\Psi_{v} v_{x} \\
& =\rho_{x}\left[\Psi_{\rho}-v \Phi_{\rho}-\frac{p^{\prime}}{\rho} \Phi_{v}\right]+v_{x}\left[\Psi_{v}-\rho \Phi_{\rho}-v \Phi_{v}\right] .
\end{aligned}
$$

Consequently, $\Phi_{t}+\Psi_{x} \equiv 0$ for all smooth solutions $(\rho, v)$ if and only if

$$
\left\{\begin{array}{l}
\Psi_{\rho}=v \Phi_{\rho}+\frac{p^{\prime}}{\rho} \Phi_{v} \\
\Psi_{v}=\rho \Phi_{\rho}+v \Phi_{v} .
\end{array}\right.
$$


We proceed further by noting $\Psi_{\rho v}=\Psi_{v \rho}$, and so $\left(v \Phi_{\rho}+\frac{p^{\prime}}{\rho} \Phi_{v}\right)_{v}=\left(\rho \Phi_{\rho}+v \Phi_{v}\right)_{\rho}$. Consequently

$$
\Phi_{\rho \rho}=\frac{p^{\prime}(\rho)}{\rho^{2}} \Phi_{v v} .
$$

In summary, $\Phi$ should solve the nonlinear wave equation (41), and we can then determine $\Psi$ from (40). We will return to these calculations in the next section.

Remark. There are many other viewpoints as to the proper "entropy formulation" for systems of conservation laws, due to Liu, to Dafermos and to others. Some of these characterize shocks as singular limits of traveling waves as a dissipative mechanism goes to zero, but even here instabilities sometimes arise. For instance, see Bertozzi-Münch-Shearer B-M-S for a physical theory of undercompressive shocks, which do not satisfy entropy conditions as above.

See also the interesting calculations in Liu-Yang [Li-Y] for an entropy functional involving two different solutions of a scalar conservation law. The presentation in $\S 6$ of Lax $[\mathrm{Lx} 2]$ is very much in the spirit of these notes.

\section{Kinetic FORMULATIONS}

(Perthame-Tadmor [P-T], Lions-Perthame-Tadmor [L-P-T1], [L-P-T2], LionsPerthame-Souganidis [L-P-S]).

7.1. A transport equation. We will next study the kinetic equation

$$
w_{t}+\mathbf{b}(y) \cdot D_{x} w=m_{y} \quad \text { in } \mathbb{R}^{n} \times \mathbb{R} \times(0, \infty),
$$

where $w=w(x, y, t)$ is the unknown, $\mathbf{b}=\mathbf{F}^{\prime}$, and $m$ is a nonnegative Radon measure on $\mathbb{R}^{n} \times \mathbb{R} \times(0, \infty)$. The derivative $m_{y}=\frac{\partial}{\partial y} m$ is understood in the distribution sense.

We also introduce, in vague analogy with Boltzmann's equation, the pseudoMaxwellian

$$
\chi_{a}(y):=\left\{\begin{aligned}
1 & \text { if } 0<y \leq a \\
-1 & \text { if } a \leq y \leq 0 \\
0 & \text { otherwise }
\end{aligned}\right.
$$

for each $a \in \mathbb{R}$.

Theorem 7. Let $w$ solve (42) for some measure $m$, as above. Assume also $w$ has the "Maxwellian" form $w=\chi_{u(x, t)}$. Then

$$
u(x, t):=\int_{\mathbb{R}} w(x, y, t) d y
$$

is an entropy solution of

$$
u_{t}+\operatorname{div} \mathbf{F}(u)=0 .
$$

Idea of proof. Let $\Phi: \mathbb{R} \rightarrow \mathbb{R}$ be convex, with $\Phi(0)=0$. Take $v \in C^{1}$ to have compact support, $v \geq 0$. We employ $v(x, t) \Phi^{\prime}(y)$ as a test function in the definition of $w$ as a weak solution of the transport equation (42):

$$
\int_{0}^{\infty} \int_{\mathbb{R}} \int_{\mathbb{R}^{n}} w\left(v \Phi^{\prime}\right)_{t}+w \mathbf{b}(y) \cdot D_{x}\left(v \Phi^{\prime}\right) d x d y d t=\int_{0}^{\infty} \int_{\mathbb{R}} \int_{\mathbb{R}^{n}}\left(v \Phi^{\prime}\right)_{y} d m .
$$


Note first of all that

$$
\int_{0}^{\infty} \int_{\mathbb{R}} \int_{\mathbb{R}^{n}} w\left(v \Phi^{\prime}\right)_{t} d x d y d t=\int_{0}^{\infty} \int_{\mathbb{R}^{n}} v_{t}\left(\int_{\mathbb{R}} w \Phi^{\prime} d y\right) d x d t .
$$

By hypothesis $w=\chi_{u(x, t)}$, and therefore if $u(x, t) \geq 0$ :

$$
\int_{\mathbb{R}} w(x, y, t) \Phi^{\prime}(y) d y=\int_{\mathbb{R}} \chi_{u(x, t)}(y) \Phi^{\prime}(y) d y=\int_{0}^{u(x, t)} \Phi^{\prime}(y) d y=\Phi(u(x, t)) .
$$

A similar computation is valid if $u(x, t) \leq 0$. Hence

$$
\int_{0}^{\infty} \int_{\mathbb{R}} \int_{\mathbb{R}^{n}} w\left(v \Phi^{\prime}\right)_{t} d x d y d t=\int_{0}^{\infty} \int_{\mathbb{R}^{n}} v_{t} \Phi(u) d x d t
$$

Likewise,

$$
\int_{0}^{\infty} \int_{\mathbb{R}} \int_{\mathbb{R}^{n}} w \mathbf{b}(y) \cdot D_{x}\left(v \Phi^{\prime}\right) d x d y d t=\int_{0}^{\infty} \int_{\mathbb{R}^{n}} D v \cdot \Psi(u) d x d t .
$$

The term on the right hand side of (45) is

$$
\int_{0}^{\infty} \int_{\mathbb{R}} \int_{\mathbb{R}^{n}}\left(v \Phi^{\prime}\right)_{y} d m=\int_{0}^{\infty} \int_{\mathbb{R}^{n}} \int_{\mathbb{R}} v \Phi^{\prime \prime} d m \geq 0,
$$

since $\Phi^{\prime \prime} \geq 0, v \geq 0$.

We conclude that

$$
\int_{0}^{\infty} \int_{\mathbb{R}^{n}} \Phi(u) v_{t}+\Psi(u) \cdot D v d x d t \geq 0
$$

for all $v$ as above, and consequently $u$ is an entropy solution of (44).

Interpretation: Since $\Phi(u)_{t}+\operatorname{div} \boldsymbol{\Psi}(u) \leq 0$ in the distribution sense, we can represent

$$
\Phi(u)_{t}+\operatorname{div} \boldsymbol{\Psi}(u)=-\mu^{\Phi}
$$

where $\mu^{\Phi}$ is a nonnegative Radon measure, depending on $\Phi$. This measure records the "change of the entropy $\Phi(u)$ across the shocks". The measure $m$ on the right hand side of the kinetic equation (42) somehow records simultaneously the information encoded in $\mu^{\Phi}$ for each entropy $\Phi$.

Remark: kinetic and level set formulations. We pause here to note that the foregoing kinetic fomulation of scalar conservation laws is, formally at least, a variant of the level set method. (Cf. Osher-Sethian [O-S].)

We generalize a bit and consider the quasilinear parabolic equation

$$
u_{t}+b_{i}(u) u_{x_{i}}-\left(a_{i j}(u) u_{x_{i}}\right)_{x_{j}}=0 \text { in } \mathbb{R}^{n} \times(0, \infty),
$$

where the symmetric matrix $\left(\left(a_{i j}\right)\right)$ is nonnegative definite.

The level set method investigates (46) by introducing a function $w=w(x, y, t)$ on $\mathbb{R}^{n+1} \times(0, \infty)$ and asking that each level set of $w$, viewed as a graph in the $y$-direction, solves (46). What PDE does $w$ then solve?

We have $w(x, u, t) \equiv c$ for some constant $c$ at $y=u=u(x, t)$ and will suppose $w_{y}<0$. Differentiating implicitly, we find

$$
\left\{\begin{array}{l}
w_{t}+w_{y} u_{t}=0, \quad w_{x_{i}}+w_{y} u_{x_{i}}=0 \quad(i=1, \ldots, n) \\
w_{x_{i} x_{j}}+w_{y x_{j}} u_{x_{i}}+w_{y x_{i}} u_{x_{j}}+w_{y y} u_{x_{i}} u_{x_{j}}+w_{y} u_{x_{i} x_{j}}=0 \quad(i, j=1, \ldots, n) .
\end{array}\right.
$$


Given that $u$ solves (46), we deduce after some calculations using (47) that

$$
0=w_{t}+b_{i} w_{x_{i}}-a_{i j} w_{x_{i} x_{j}}+2 \frac{a_{i j} w_{y x_{i}} w_{x_{j}}}{w_{y}}+-\frac{a_{i j} w_{y y} w_{x_{i}} w_{x_{j}}}{w_{y^{2}}}+\frac{a_{i j}^{\prime} w_{x_{i}} w_{x_{j}}}{w_{y}} .
$$

Hence, setting $u=y$ in the arguments of $b_{i}, a_{i j}$, we derive the kinetic formulation

$$
w_{t}+b_{i}(y) w_{x_{i}}-a_{i j}(y) w_{x_{i} x_{j}}=m_{y}
$$

for

$$
m:=-\frac{a_{i j}(y) w_{x_{i}} w_{x_{j}}}{w_{y}} .
$$

We note finally that $m \geq 0$, since $w_{y}<0$.

7.2. Application: a hydrodynamical limit. Consider the scaled transport equation

$$
w_{t}^{\varepsilon}+\mathbf{b}(y) \cdot D_{x} w^{\varepsilon}=\frac{1}{\varepsilon}\left(\chi_{u^{\varepsilon}}-w^{\varepsilon}\right)
$$

for

$$
u^{\varepsilon}(x, t):=\int_{\mathbb{R}} w^{\varepsilon}(x, y, t) d y .
$$

Theorem 8. As $\varepsilon \rightarrow 0$, we have $w^{\varepsilon} \rightarrow w$ weakly $*$ in $L^{\infty}$, where $w=\chi_{u}$ and

$$
w_{t}+\mathbf{b}(y) \cdot D_{x} w=m_{y} \quad \text { in } \mathbb{R}^{n} \times \mathbb{R} \times(0, \infty)
$$

for some nonnegative Radon measure $m$. Also, $u$ is a unique entropy solution of

$$
u_{t}+\operatorname{div} \mathbf{F}(u)=0 \quad \text { on } \mathbb{R}^{n} \times(0, \infty) .
$$

Idea of proof. We show that we can write

$$
\frac{1}{\varepsilon}\left(\chi_{u^{\varepsilon}}-w^{\varepsilon}\right)=m_{y}^{\varepsilon}
$$

for some nonnegative function $m^{\varepsilon}$. We then extract a sequence $\varepsilon_{r} \rightarrow 0$, so that

$$
w_{t}+\mathbf{b}(y) \cdot D_{x} w=m_{y}
$$

in the weak sense, $m$ a measure. Since $\chi_{u^{\varepsilon}}-w^{\varepsilon}=\varepsilon m_{y}^{\varepsilon}, \chi_{u^{\varepsilon}} \rightarrow w$ weakly $*$ in $L^{\infty}$, and in fact $w=\chi_{u}$. So according to the kinetic formulation, $u$ solves the conservation law (49).

7.3. Kinetic formulation of Euler's equations. Let us return to the compressible Euler equations, with the explicit equation of state

$$
p(\rho)=\kappa \rho^{\gamma}, \quad \text { where } \kappa=\frac{(\gamma-1)^{2}}{4 \gamma}, \gamma>1,
$$

the constant $\kappa$ so selected to simplify the algebra. We continue from $\S 6.3$ some calculations for entropy functions:

Theorem 9. (i) The solution of (41) with initial conditions $\Phi=0, \Phi_{\rho}=\delta_{0}$, the Dirac mass at the origin, is

$$
\chi(\rho, v)=\left(\rho^{\gamma-1}-v^{2}\right)_{+}^{\lambda}, \quad \lambda=\frac{3-\gamma}{2(\gamma-1)} .
$$

The general solution of (41) with initial conditions $\Phi=0, \Phi_{\rho}=g$ is

$$
\Phi(\rho, v)=\int_{\mathbb{R}} g(y) \chi(\rho, y-v) d y .
$$


(ii) Furthermore, $\Phi$ is convex in $(\rho, \rho v)$ if and only if $g$ is convex. The entropy flux $\Psi$ associated with $\Phi$ is

$$
\Psi(\rho, v)=\int_{\mathbb{R}} g(y)(\theta y+(1-\theta) v) \chi(\rho, y-v) d y
$$

for $\theta=\frac{\gamma-1}{2}$.

See [L-P-T2] for proof. We can regard $\chi$ as a sort of pseudo-Maxwellian, parameterized by the macroscopic parameters $\rho, v$.

Theorem 10. Suppose $\rho \geq 0$ a.e. Then $(\rho, \rho v)$ is an entropy solution of Euler's equations if and only if there exists a nonpositive measure $m$ on $\mathbb{R} \times \mathbb{R} \times(0, \infty)$ such that

$$
w:=\chi(\rho, y-v)
$$

satisfies

$$
w_{t}+[(\theta y+(1-\theta) v) w]_{x}=m_{y y} .
$$

We call (50), (151) a kinetic formulation of Euler's equation.

Idea of proof. Define the distributions

$$
T:=w_{t}+[(\theta y+(1-\theta) v) w]_{x}, \frac{\partial^{2} M}{\partial y^{2}}:=T .
$$

Take $\Phi, \Psi$ to be an entropy/entropy flux pair as above. Then

$$
\Phi_{t}+\Psi_{x}=\int_{\mathbb{R}} g(y)\left(w_{t}+[(\theta y+(1-\theta) v) w]_{x}\right) d y .
$$

Suppose now $\phi(x, y, t)=\alpha(x, t) \beta(y)$, where $\alpha, \beta \geq 0$ are smooth, with compact support. Take $g$ so that $g^{\prime \prime}=\beta$. Then

$$
\begin{aligned}
-\int_{0}^{\infty} \int_{\mathbb{R}} \Phi \alpha_{t}+\Phi \alpha_{x} d x d t & =\int_{0}^{\infty} \int_{\mathbb{R}} \int_{\mathbb{R}} \alpha g\left(w_{t}+[(\theta y+(1-\theta) v) w]_{x}\right) d x d y d t \\
& =\langle T, \alpha g\rangle=\langle M, \alpha \beta\rangle=\langle M, \phi\rangle .
\end{aligned}
$$

Now if $(\rho, \rho v)$ is an entropy solution, then

$$
\int_{0}^{\infty} \int_{\mathbb{R}} \Phi \alpha_{t}+\Psi \alpha_{x} d x d t \geq 0
$$

since $\alpha \geq 0$, and consequently $\langle M, \phi\rangle \leq 0$. Thus $M$ is represented by a nonpositive measure.

Perthame's book $[\mathrm{P}$ provides a good overview of kinetic formulations of nonlinear PDE.

\section{Hysteresis IN PHASE TRANSITIONS}

(Novick Cohen-Pego [NC-P], Plotnikov [P], [E-P]).

This section discusses how some entropy-like calculations let us sometimes understand the effects of a regularization for an ill-posed diffusion equation. 


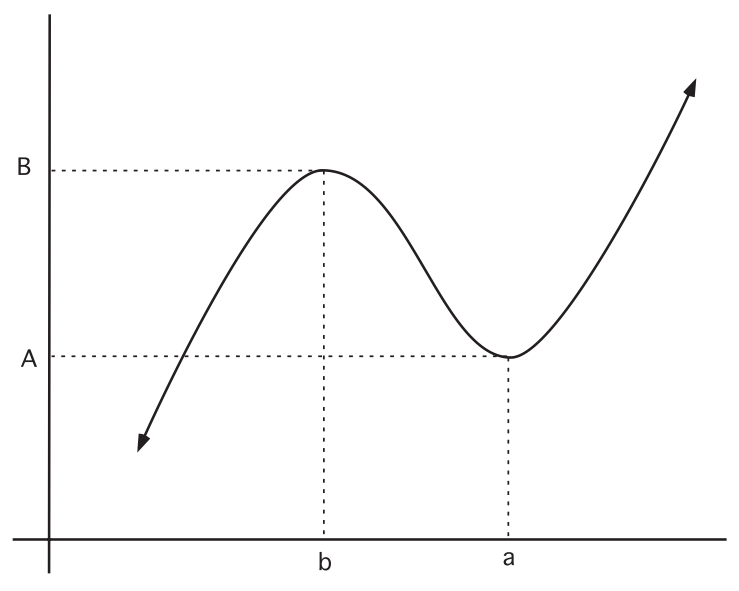

FiguRE 3.

8.1. An ill-posed problem. We turn next to the nonlinear diffusion equation

$$
u_{t}=\Delta \phi(u)
$$

where the nonlinearity $\phi$ has the cubic-type structure illustrated in Figure 3 Our PDE is ill-posed forwards in time whenever $u \in(b, a)$. So consider instead this "viscous" regularization for $\varepsilon>0$ and $U$ a smooth, bounded domain in $\mathbb{R}^{n}$ :

$$
\begin{cases}u_{t}^{\varepsilon}=\Delta \phi\left(u^{\varepsilon}\right)+\varepsilon \Delta u_{t}^{\varepsilon} & \text { in } U \times(0, \infty) \\ \frac{\partial}{\partial \nu}\left(\phi\left(u^{\varepsilon}\right)+\varepsilon u_{t}^{\varepsilon}\right)=0 & \text { on } \partial U \times(0, \infty) \\ u^{\varepsilon}=u_{0}^{\varepsilon} & \text { on } U \times\{t=0\} .\end{cases}
$$

Introduce the new unknown function

$$
v^{\varepsilon}:=\phi\left(u^{\varepsilon}\right)+\varepsilon u_{t}^{\varepsilon}
$$

then

$$
\left\{\begin{array}{l}
u_{t}^{\varepsilon}=\frac{v^{\varepsilon}-\phi\left(u^{\varepsilon}\right)}{\varepsilon} \\
v^{\varepsilon}-\varepsilon \Delta v^{\varepsilon}=\phi\left(u^{\varepsilon}\right)
\end{array}\right.
$$

with Neumann boundary conditions for $v^{\varepsilon}$.

8.2. Estimates, weak convergence. We have $\sup \left|u^{\varepsilon}, v^{\varepsilon}\right| \leq C$ for some constant $C$. Next, take $g: \mathbb{R} \rightarrow \mathbb{R}$ to be nondecreasing, and set

$$
G^{\prime}(z)=g(\phi(z)) .
$$

We compute using (53), (54) that

$$
G\left(u^{\varepsilon}\right)_{t}=\operatorname{div}\left(g\left(v^{\varepsilon}\right) D v^{\varepsilon}\right)-g^{\prime}\left(v^{\varepsilon}\right)\left|D v^{\varepsilon}\right|^{2}-\left(g\left(v^{\varepsilon}\right)-g\left(\phi\left(u^{\varepsilon}\right)\right)\right)\left(\frac{v^{\varepsilon}-\phi\left(u^{\varepsilon}\right)}{\varepsilon}\right),
$$

the last two terms being nonnegative. The point is that this is somewhat like an entropy/entropy flux calculation for conservation laws, although the relevant PDE are quite different. 


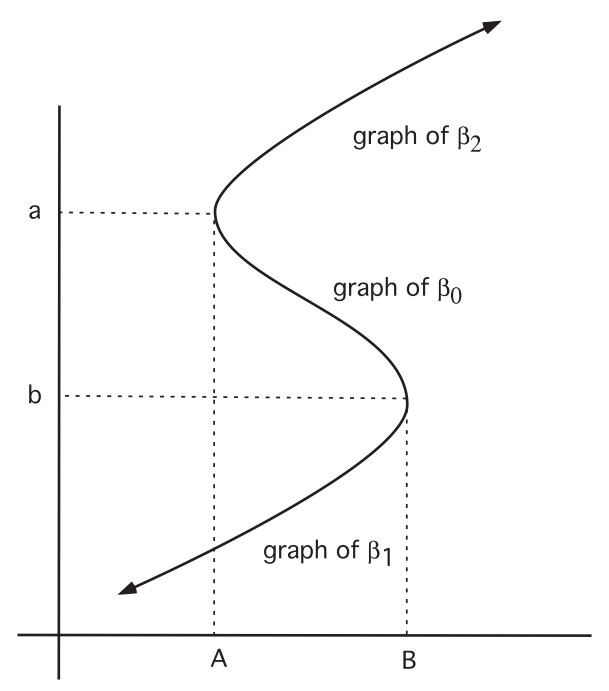

FIGURE 4.

Take a sequence $\varepsilon_{j} \rightarrow 0$ such that $u^{\varepsilon_{j}}, v^{\varepsilon_{j}} \rightarrow u, v$ weakly $*$ in $L^{\infty}$. The goal is understanding the relationships between $u, v$, and the equations they satisfy. First, we introduce the three branches $\beta_{i}(i=0,1,2)$ of $\phi^{-1}$ in Figure 4.

In a very interesting paper $[\mathrm{P}$, Plotnikov has shown

Theorem 11. There exist measurable functions $\lambda_{0}, \lambda_{1}, \lambda_{2}$ such that

(i) $0 \leq \lambda_{i} \leq 1, \quad \sum_{i=0}^{2} \lambda_{i}=1$.

(ii) Furthermore,

$$
F\left(u^{\varepsilon_{j}}\right) \rightarrow \bar{F}:=\sum_{i=0}^{2} \lambda_{i} F\left(\beta_{i}(v)\right)
$$

weakly $*$ in $L^{\infty}$, for each continuous function $F$.

(iii) We also have $v^{\varepsilon_{j}}, \phi\left(u^{\varepsilon_{j}}\right) \rightarrow v$ strongly in $L^{2}$.

Passing to limits as $\varepsilon=\varepsilon_{j} \rightarrow 0$ in (55), we conclude that

$$
\bar{G}_{t}-\operatorname{div}(g(v) D v) \leq-g^{\prime}(v)|D v|^{2}
$$

for each nondecreasing $g$ as above. Similarly

$$
u_{t}=\Delta v \text {. }
$$

8.3. A free boundary problem with hysteresis. Suppose now that $\lambda_{0} \equiv 0$, $\lambda_{1} \equiv 1$ in $V_{1}, \lambda_{2} \equiv 1$ in $V_{2}$, where $V_{1}, V_{2}$ are two open regions, with a smooth interface $\Gamma:=\bar{V}_{1} \cap \bar{V}_{2}$. We assume that $u, v$ are smooth in $\bar{V}_{1}, \bar{V}_{2}$, and write $u_{i}, v_{i}$ to denote the values along $\Gamma$.

We want to understand how $\Gamma$ moves. Let $\left(\nu^{1}, \ldots, \nu^{n}, \nu^{n+1}\right)=\left(\nu, \nu^{n+1}\right)$ denote the unit normal along $\Gamma$ pointing into $V_{1}$.

Theorem 12. (i) We have

$$
\begin{cases}\beta_{1}(v)_{t}=\Delta v & \text { in } V_{1} \\ \beta_{2}(v)_{t}=\Delta v & \text { in } V_{2}\end{cases}
$$




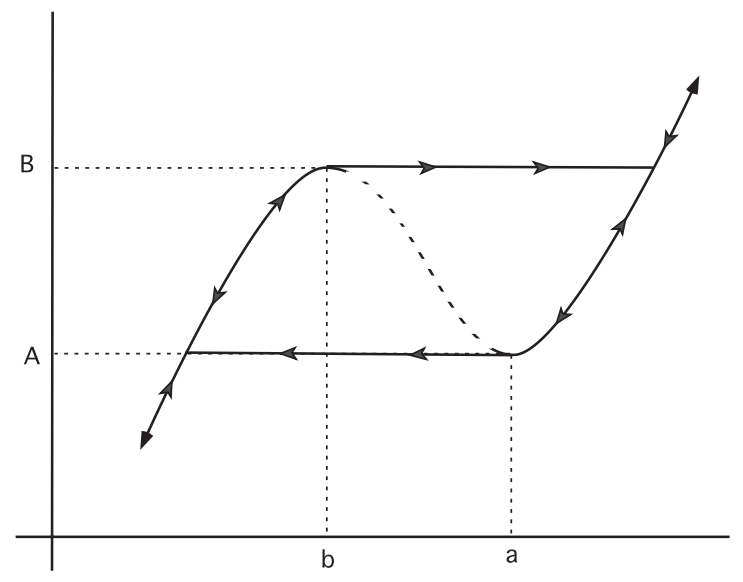

FiguRE 5.

(ii) Furthermore,

$$
v_{1}=v_{2} \quad \text { and } \quad \nu^{n+1}[u]=\nu \cdot\left[D_{x} v\right] \quad \text { along } \Gamma,
$$

where $[u]:=u_{1}-u_{2},\left[D_{x} v\right]:=D_{x} v_{1}-D_{x} v_{2}$.

(iii) Also,

$$
\begin{cases}\nu^{n+1}=0 & \text { if } v \neq A, B \\ \nu^{n+1} \geq 0 & \text { if } v=A \\ \nu^{n+1} \leq 0 & \text { if } v=B,\end{cases}
$$

where we write $v=v^{1}=v^{2}$ along $\Gamma$.

Statement (iii) says that the nonlinearity $\phi$ generates a hysteresis loop, illustrated in Figure 5, which we interpret as a "supercooled" Stefan problem with phase transition between the temperatures $A$ and $B$. See Visintin $\mathrm{Vs}$ for more about hysteresis effects in PDE.

Idea of proof. We have

$$
\bar{G}= \begin{cases}G\left(\beta_{1}(v)\right) & \text { in } V_{1} \\ G\left(\beta_{2}(v)\right) & \text { in } V_{2},\end{cases}
$$

for each function $G$ as above. In particular,

$$
u= \begin{cases}\beta_{1}(v) & \text { in } V_{1} \\ \beta_{2}(v) & \text { in } V_{2},\end{cases}
$$

and so (58) follows from (57). Also, our integrating by parts using (57) gives the Rankine-Hugoniot relation (59).

We next multiply (56) by a nonnegative function $\zeta \in C_{c}^{\infty}$ and integrate by parts to find

$$
\begin{aligned}
& 0 \geq \iint_{V_{1}} g(v)\left(\beta_{1}(v)_{t}-\Delta v\right) \zeta d x d t \\
& \quad+\iint_{V_{2}} g(v)\left(\beta_{2}(v)_{t}-\Delta v\right) \zeta d x d t+\int_{\Gamma}\left(\nu^{n+1}[G(u)]-\nu \cdot\left[D_{x} v\right] g(v)\right) \zeta d \mathcal{H}^{n} .
\end{aligned}
$$


Consequently $\nu^{n+1}[G(u)]-\nu \cdot\left[D_{x} v\right] g(v) \leq 0$ along $\Gamma$, and so (59) implies $\nu^{n+1}([G(u)]-g(v)[u]) \leq 0$ for each nondecreasing function $g$. Since $G^{\prime}(z)=g(\phi(z))$, this says

$$
\nu^{n+1}\left(\int_{\beta_{1}(v)}^{\beta_{2}(v)} g(\phi(s))-g(v) d s\right) \geq 0 \quad \text { along } \Gamma .
$$

If $A<v<B$, we first take $g^{+}$to be zero on $(-\infty, v]$, positive and nondecreasing on $(v, \infty)$. Then

$$
\int_{\beta_{1}(v)}^{\beta_{2}(v)} g^{+}(\phi(s))-g^{+}(v) d s>0
$$

and so $\nu^{n+1} \geq 0$. Next select $g^{-}$to be negative and nondecreasing on $(-\infty, v)$, zero on $[v, \infty)$. This forces

$$
\int_{\beta_{1}(v)}^{\beta_{2}(v)} g^{-}(\phi(s))-g^{-}(v) d s<0 ;
$$

whence $\nu^{n+1} \leq 0$. Consequently $\nu^{n+1}=0$ if $A<v<B$. If $v=A$, we take $g^{+}$as above, to deduce $\nu^{n+1} \geq 0$. Likewise, $\nu^{n+1} \leq 0$ if $v=B$.

\section{HAMILTON-JACOBI EQUATIONS}

(Crandall-Lions [C-L, C-E-L).

Many first-order PDE are structurally quite different from conservation laws, and yet these too sometimes admit weak interpretations involving dissipation effects. The trick is to switch our viewpoint from integral formulas to pointwise ones.

9.1. Viscosity solutions. A PDE of the form

$$
u_{t}+H(D u)=0 \quad \text { in } \mathbb{R}^{n} \times(0, \infty)
$$

is called a Hamilton-Jacobi equation. The unknown is $u$ and the Hamiltonian $H$ is given. As before, $D u=\left(u_{x_{1}}, \ldots, u_{x_{n}}\right)$.

Definition. A bounded uniformly continuous function $u$ is called a viscosity solution of (61) provided for each $v \in C^{\infty}$,

$$
\left\{\begin{array}{l}
\text { if } u-v \text { has a local maximum (resp. minimum) at a } \\
\text { point }\left(x_{0}, t_{0}\right) \in \mathbb{R}^{n} \times(0, \infty), \\
\text { then } v_{t}\left(x_{0}, t_{0}\right)+H\left(D v\left(x_{0}, t_{0}\right)\right) \leq 0(\text { resp. } \geq 0) .
\end{array}\right.
$$

Motivation. As before we can motivate the definition by the vanishing viscosity method, and this procedure accounts for the name 1 So consider the regularized PDE

$$
u_{t}^{\varepsilon}+H\left(D u^{\varepsilon}\right)=\varepsilon \Delta u^{\varepsilon} .
$$

It is instructive to check that $u$ is a viscosity solution of (61), when $u^{\varepsilon} \rightarrow u$ locally uniformly.

\footnotetext{
${ }^{1}$ In fact, Crandall and Lions originally considered the name "entropy solutions".
} 
9.2. A cautionary example. There are formal mathematical connections at the level of PDE between thermodynamics and mechanics, as explained for instance in Peterson $[\mathrm{Pe}$. For instance, the Clausius-Clapeyron condition for phase transitions is just the Rankine-Hugoniot condition, as before. However, we must be very careful when considering nonsmooth solutions, as this example, found with D. Ostrov, shows.

The van der Waals equation of state is

$$
F(V, P, T):=P-\frac{R T}{V-b}+\frac{a}{V^{2}}=0 .
$$

We seek $G=G(T, P)$ satisfying this, where $\frac{\partial G}{\partial T}=-S, \frac{\partial G}{\partial P}=V$ according to (12). We can think of $T=T_{0}$ as a fixed parameter, and so regard (63) as the implicit ODE

$$
F\left(\frac{\partial G}{\partial P}, P, T_{0}\right)=0
$$

For certain values of the parameters $a, b, T_{0}$, the level set $\left\{F\left(\cdot, \cdot, T_{0}\right)=0\right\}$ has the cubic shape in Figure 6

A standard thermodynamic construction yields a concave solution $G$ with a discontinuity in its derivative occurring at the Maxwell equal area point $P_{0}$, as illustrated in Figure 7 .

However, $G$ is not a viscosity solution of (64). To see this, notice that we can touch the graph of $G$ from above at the point $P_{0}$ by a smooth function $v$, with $v^{\prime}\left(P_{0}\right)$ taking any value between $V_{l}$ and $V_{r}$. If $G$ were a viscosity solution of (64), it would follow that

$$
F\left(V, P_{0}, T_{0}\right) \leq 0 \text { for all } V_{r} \leq V \leq V_{l} .
$$

But this is not so, since $F$ changes sign across the curve.

Remark. The viscosity solution interpretation of the ODE (64) in effect predicts a sort of hysteresis loop behavior, as drawn in Figure 8

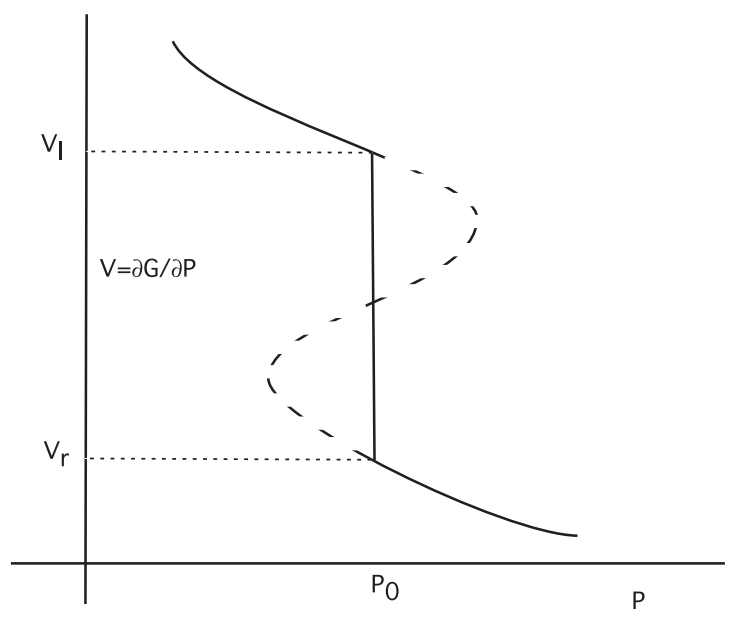

FIGURE 6. 


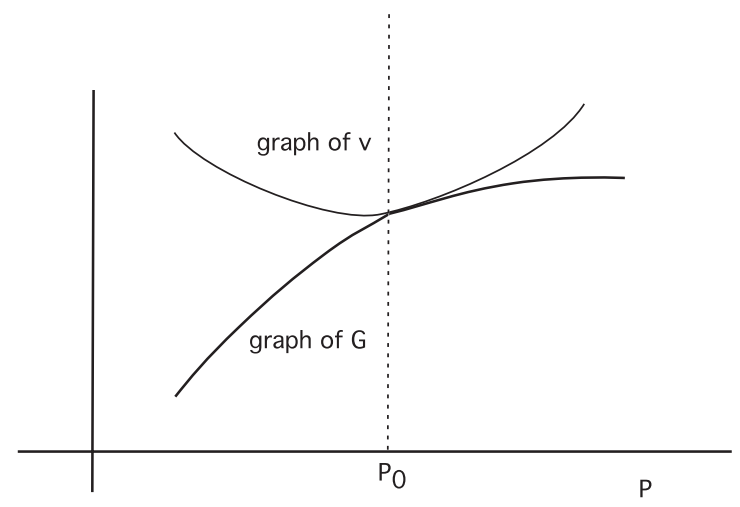

FIGURE 7.

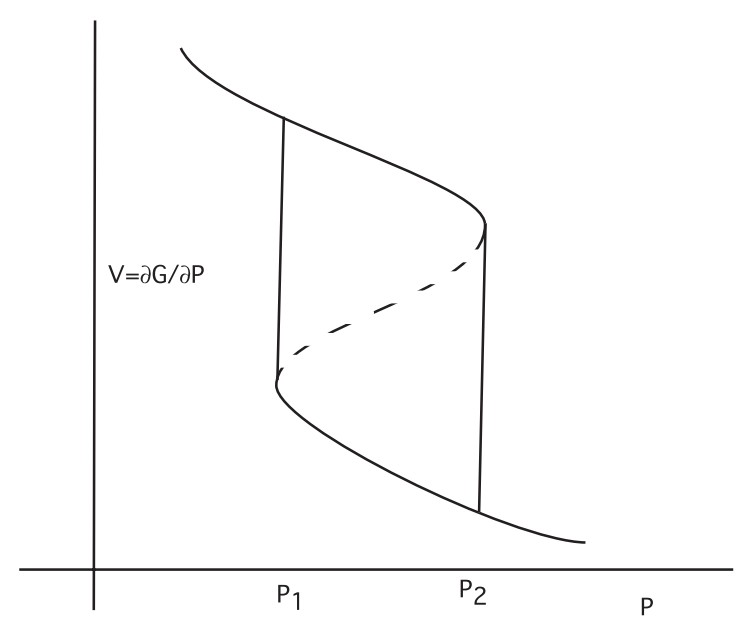

FiguRE 8.

Under the assumption that $F<0$ to the left of the curve and $F>0$ to the right, an upward pointing corner in $G$ can occur only for $P=P_{1}$. A downward corner in $G$ can occur only for $P=P_{2}$, although on physical grounds the Gibbs potential $G$ should be concave and thus not have any downward pointing corners. (Cf. Oleinik's condition (36), (37).)

9.3. A diffusion limit. (E2]) The next example shows how we can sometimes demonstrate dissipative effects in singular scaling limits. We introduce for each $\varepsilon>0$ a coupled linear first-order transport PDE:

$$
\left\{\begin{aligned}
w_{t}^{k, \varepsilon}+\frac{1}{\varepsilon} b^{k} \cdot D w^{k, \varepsilon} & =\frac{1}{\varepsilon^{2}} \sum_{l=1}^{m} c_{k l} w^{l, \varepsilon} & & \text { in } \mathbb{R}^{n} \times(0, \infty) \\
w^{k, \varepsilon} & =g & & \text { on } \mathbb{R}^{n} \times\{t=0\}
\end{aligned}\right.
$$

for $k=1, \ldots, m$. The unknown is $\mathbf{w}^{\varepsilon}=\left(w^{1, \varepsilon}, \ldots, w^{m, \varepsilon}\right)$. We are given the matrix $C=\left(\left(c_{k l}\right)\right)_{m \times m}$ and the velocity vectors $\left\{b^{k}\right\}_{k=1}^{m}$ in $\mathbb{R}^{n}$. 
The left hand side of (66) is for each $k$ a linear, constant coefficient transport operator, and the right hand side of (66) represents linear coupling. What happens as $\varepsilon \rightarrow 0$ ?

Let us assume:

$$
c_{k l}>0 \quad \text { if } k \neq l, \quad \sum_{l=1}^{m} c_{k l}=0 .
$$

Then there exists a unique vector $\pi=\left(\pi_{1}, \ldots, \pi_{m}\right)$ satisfying

$$
\pi_{k}>0(k=1, \ldots, m), \quad \sum_{k=1}^{m} \pi_{k}=1, \quad \sum_{k=1}^{m} c_{k l} \pi_{k}=0 .
$$

We make the additional assumption of average velocity balance:

$$
\sum_{k=1}^{m} \pi_{k} b^{k}=0
$$

- Construction of diffusion coefficients. Write $\mathbb{1}:=(1, \ldots, 1) \in \mathbb{R}^{m}$. Then (67), (68) and Perron-Frobenius theory assert that $\mathbb{1 1}$ spans the nullspace of $C$ and $\pi$ spans the nullspace of $C^{*}$. In view of (69), for each $j \in\{1, \ldots, n\}$ the vector $b_{j}:=\left(b_{j}^{1}, \ldots, b_{j}^{m}\right) \in \mathbb{R}^{m}$ is perpendicular to the nullspace of $C^{*}$ and thus lies in the range of $C$. There consequently exists a unique vector $d_{j} \in \mathbb{R}^{m}$ solving

$$
C d_{j}=-b_{j},
$$

normalized by our requiring $d_{j} \cdot \mathbb{1}=0$. We write $d_{j}=\left(d_{j}^{1}, \ldots, d_{j}^{m}\right)$, and then define the diffusion coefficients

$$
a^{i j}:=\sum_{k=1}^{m} \pi_{k} b_{i}^{k} d_{j}^{k}
$$

It is an exercise to check that the matrix $\left(\left(a^{i j}\right)\right)$ is nonnegative definite.

Theorem 13. As $\varepsilon \rightarrow 0$, we have $w^{k, \varepsilon} \rightarrow u$ locally uniformly, where $u$ solves the diffusion equation

$$
u_{t}-\sum_{i, j=1}^{n} a^{i j} u_{x_{i} x_{j}}=0 .
$$

Idea of proof. We can find a subsequence $\varepsilon_{r} \rightarrow 0$ such that $\mathbf{w}^{\varepsilon_{r}} \rightarrow \mathbf{w}=u \mathbb{1}$, locally uniformly, for some scalar function $u=u(x, t)$.

We assert that $u$ is a viscosity solution of (71). This means that if $v \in C^{2}$ and

$$
\left\{\begin{array}{l}
u-v \text { has a local maximum (resp. minimum) at } \\
\text { a point }\left(x_{0}, t_{0}\right) \in \mathbb{R}^{n} \times(0, \infty)
\end{array}\right.
$$

then

$$
v_{t}\left(x_{0}, t_{0}\right)-\sum_{i, j=1}^{n} a^{i j} v_{x_{i} x_{j}}\left(x_{0}, t_{0}\right) \leq 0(\text { resp. } \geq 0) .
$$

To prove this, let us suppose $u-v$ has a strict local maximum at some point $\left(x_{0}, t_{0}\right)$. Define then the perturbed test functions $\mathbf{v}^{\varepsilon}:=\left(v^{1, \varepsilon}, \ldots, v^{m, \varepsilon}\right)$, where

$$
v^{k, \varepsilon}:=v-\varepsilon \sum_{j=1}^{n} d_{j}^{k} v_{x_{j}},
$$


the constants $d_{j}^{k}$ satisfying (70). Then $w^{k, \varepsilon}-v^{k, \varepsilon}$ has a local maximum at a point $\left(x_{\varepsilon}^{k}, t_{\varepsilon}^{k}\right)$, and $\left(x_{\varepsilon}^{k}, t_{\varepsilon}^{k}\right) \rightarrow\left(x_{0}, t_{0}\right)$ as $\varepsilon=\varepsilon_{r} \rightarrow 0$.

We then employ the transport PDE (66) and various algebraic relations above to eliminate the terms of order $\frac{1}{\varepsilon}, \frac{1}{\varepsilon^{2}}$ and thereby to deduce:

$$
v_{t}\left(x_{0}, t_{0}\right)-\sum_{i, j=1}^{n} \underbrace{\left.\sum_{k=1}^{n} \pi_{k} b_{i}^{k} d_{j}^{k}\right)}_{a^{i j}} v_{x_{i} x_{j}}\left(x_{0}, t_{0}\right) \leq o(1) .
$$

See [E2] for details. A similar argument provides the opposite inequality should $u-v$ have a minimum at $\left(x_{0}, t_{0}\right)$.

- See Pinsky $[\mathrm{P}$ for other techniques, based upon interpreting (66) as a random evolution. The system of PDE (66) is reversible in time and yet the diffusion equation (71) is not. Exercise for the reader: Where did the irreversibility come from?

\section{LARGE DEVIATIONS}

(Varadhan [V], Dembo-Zeitouni [D-Z]).

10.1. Background. Let $\left\{P_{n}\right\}_{n=1}^{\infty}$ be a family of Borel probability measures on a separable, complete, metric space $\Sigma$.

We say that $\left\{P_{n}\right\}_{n=1}^{\infty}$ satisfies the large deviation principle with rate function $I: \Sigma \rightarrow \mathbb{R}$ provided:

$$
\begin{cases}\lim \sup _{n \rightarrow \infty} \frac{1}{n} \log P_{n}(C) \leq-\inf _{C} I & (C \text { closed }) \\ & \liminf \inf _{n \rightarrow \infty} \frac{1}{n} \log P_{n}(U) \geq-\inf _{U} I \quad(U \text { open }) .\end{cases}
$$

The rate function $I$ is called the entropy function in the book of Ellis El, which contains clear explanations of the connections with statistical mechanics and thermodynamics.

10.2. Cramer's Theorem. Let $(\Omega, \mathcal{F}, \pi)$ be a probability space and suppose $\mathbf{Y}_{k}$ : $\Omega \rightarrow \mathbb{R}^{m}(k=1, \ldots)$ are independent, identically distributed random variables. Write $\mathbf{Y}:=\mathbf{Y}_{1}$. We will study the partial sums

$$
\mathbf{S}_{n}:=\frac{\mathbf{Y}_{1}+\cdots+\mathbf{Y}_{n}}{n}
$$

and their distributions $P_{n}$ on $\Sigma=\mathbb{R}^{m}$.

Define

$$
F(p):=\log E\left(e^{p \cdot \mathbf{Y}}\right)=\log \left(\int_{\Omega} e^{p \cdot \mathbf{Y}} d \pi\right),
$$

and introduce as in (11) the Legendre transform of $F$ :

$$
L(q)=\sup _{p \in \mathbb{R}^{m}}(p \cdot q-F(p)) .
$$

Cramer's Theorem asserts this to be a large deviation rate function:

Theorem 14. The probability measures $\left\{P_{n}\right\}_{n=1}^{\infty}$ satisfy a large deviation principle with rate function $I(\cdot)=L(\cdot)$. 
Idea of proof. Following ideas of R. Jensen, we will use PDE methods to prove for each nice function $g$ that

$$
\lim _{n \rightarrow \infty} \frac{1}{n} \log \left(\int_{\mathbb{R}^{m}} e^{n g} d P_{n}\right)=\sup _{\mathbb{R}^{m}}(g-L) .
$$

This implies that $L$ is the rate function.

We fix any point $x \in \mathbb{R}^{m}$ and then write $t_{k}:=k / n$. We define also

$$
w_{n}\left(x, t_{k}\right):=E\left(h_{n}\left(\frac{\mathbf{Y}_{1}+\cdots+\mathbf{Y}_{k}}{n}+x\right)\right) \text {, }
$$

where $h_{n}:=e^{n g}$. Finally, set

$$
u_{n}\left(x, t_{k}\right):=\frac{1}{n} \log w_{n}\left(x, t_{k}\right) .
$$

Extend $u_{n}(x, t)$ to be linear in $t$ for $t \in\left[t_{k}, t_{k+1}\right]$. Then there exists a sequence $n_{r} \rightarrow \infty$ such that $u_{n_{r}} \rightarrow u$ locally uniformly.

We assert that $u$ is a viscosity solution of the PDE

$$
u_{t}-F(D u)=0 .
$$

To verify this, we take any $v \in C^{2}$ and suppose $u-v$ has a strict maximum at a point $\left(x_{0}, t_{0}\right)$. We must prove:

$$
v_{t}\left(x_{0}, t_{0}\right)-F\left(D v\left(x_{0}, t_{0}\right)\right) \leq 0 .
$$

We can find for each index $n=n_{r}$ points $\left(x_{n}, t_{k_{n}}\right)$ such that

$$
u_{n}\left(x_{n}, t_{k_{n}}\right)-v\left(x_{n}, t_{k_{n}}\right)=\max _{x \in \mathbb{R}^{m}, k=0, \ldots}\left[u_{n}\left(x, t_{k}\right)-v\left(x, t_{k}\right)\right]
$$

and $\left(x_{n}, t_{k_{n}}\right) \rightarrow\left(x_{0}, t_{0}\right)$ as $n=n_{r} \rightarrow \infty$. We calculate that

$$
\frac{v\left(x_{n}, t_{k_{n}}\right)-v\left(x_{n}, t_{k_{n}-1}\right)}{1 / n} \leq \log E\left(e^{D v\left(x_{n}, t_{k_{n}-1}\right) \cdot \mathbf{Y}+\beta_{n}}\right),
$$

for a small error term $\beta_{n}$. Pass to limits:

$$
v_{t}\left(x_{0}, t_{0}\right) \leq \log E\left(e^{D v\left(x_{0}, t_{0}\right) \cdot \mathbf{Y}}\right)=F\left(D v\left(x_{0}, t_{0}\right)\right) .
$$

This is (74), and the reverse inequality likewise holds should $u-v$ have a strict local minimum at a point $\left(x_{0}, t_{0}\right)$.

So $u$ is a viscosity solution of (73), and we can invoke the explicit Hopf-Law formula (cf. [E1]):

In particular

$$
u(x, t)=\sup _{y}\left\{g(y)-t L\left(\frac{y-x}{t}\right)\right\} .
$$

$$
u(0,1)=\sup _{y}\{g(y)-L(y)\}
$$

But

$$
\begin{aligned}
u_{n}(0,1) & =\frac{1}{n} \log w_{n}\left(0, t_{n}\right)=\frac{1}{n} \log E\left(h_{n}\left(\frac{\mathbf{Y}_{1}+\cdots+\mathbf{Y}_{n}}{n}\right)\right) \\
& =\frac{1}{n} \log E\left(e^{n g\left(\mathbf{S}_{n}\right)}\right)=\frac{1}{n} \log \left(\int_{\mathbb{R}^{m}} e^{n g} d P_{n}\right) .
\end{aligned}
$$

As $u_{n}(0,1) \rightarrow u(0,1)$, this and (75) confirm the limit (72).

This proof illustrates the vague principle that rate functions, interpreted as functions of appropriate parameters, are viscosity solutions of Hamilton-Jacobi type 
PDE. The general validity of this principle is unclear, but there are certainly many instances in the literature, for instance Freidlin-Wentzell [F-W].

\section{SOME FURTHER TOPICS}

11.1. Decay to equilibrium. There has been great recent interest in "entropy" techniques for deriving decay rate estimates as $t \rightarrow \infty$ for nonlinear parabolic PDE, having for instance the form

$$
u_{t}=\operatorname{div}(D u+u D V)
$$

In this case $u_{\infty}:=e^{-V}$ is an equilibrium, and the dynamics can be rewritten as

$$
u_{t}=\operatorname{div}\left(u_{\infty} D\left(\frac{u}{u_{\infty}}\right)\right) .
$$

See Carrillo et al. C-J-M] for clever differential inequality calculations. Villani's survey [Vi] on Monge-Kantorovich mass transfer methods examines similar issues.

11.2. Equilibria of Euler's equations. Several authors have introduced statistical mechanics, maximum entropy principles to derive semilinear elliptic PDE describing equilibrium states for two-dimensional inviscid fluids. Large deviation arguments provide some mathematical justification. See, for instance, BoucherEllis-Turkington [B-E-T], Lions [L], Mikelic-Robert [M-R], Turkington [Tu], and also Chapter 7 of the book [M-P] of Marchioro and Pulvirenti. DiBattista, Haven, Majda and Turkington [D-H-M-T provide a related model of Jupiter's atmosphere.

Freidlin [F] presents an extremely interesting alternative approach.

\section{REFERENCES}

[AJP] American Journal of Physics, Theme issue on Thermal and Statistical Physics, 67, December, 1999.

[B-M-S] A. Bertozzi, A. Münch and M. Shearer, Undercompressive waves in driven thin film flow: theory, computation, and experiment, in Trends in Mathematical Physics AMS/IP Stud. Adv. Math, AMS, 1999. MR 1708749

[B-T] S. Bharatha and C. Truesdell, Classical Thermodynamics as a Theory of Heat Engines, Springer, 1977. MR 0471650 (57:11375)

[B-E-T] C. Boucher, R. Ellis and B. Turkington, Derivation of maximum entropy principles in two-dimensional turbulence via large deviations, J. Stat. Physics 98 (2000), 12351278. MR 1751700 (2001a:76087)

[B] K. Brakke, The Motion of a Surface by its Mean Curvature, Mathematical Notes 20, Princeton University Press, 1978. MR 0485012 (82c:49035)

[C-K-N] L. Caffarelli, R. Kohn and L. Nirenberg, Partial regularity of suitable weak solutions of the Navier-Stokes equations, Comm. Pure Appl. Math. 35 (1982), 771-831. MR 0673830 (84m:35097)

[C] H. Callen, Thermodynamics and an Introduction to Thermostatistics (2nd ed.), Wiley, 1985.

[C-J-M] J. Carrillo, A. Jungel, P. Markowich, G. Toscani and A. Unterreiter, Entropy dissipation methods for degenerate parabolic problems and generalized Sobolev inequalities, Monatsh. Math. 133 (2001), 1-82. MR 1853037 (2002j:35188)

[C-N] B. Coleman and W. Noll, The thermodynamics of elastic materials with heat conduction and viscosity, Arch. Rat. Mech. Analysis 13 (1963), 167-178. MR $0153153(27: 3122)$

[C-O-S] B. Coleman, D. Owen and J. Serrin, The second law of thermodynamics for systems with approximate cycles, Arch. Rat. Mech. Analysis 77 (1981), 103-142. MR 0633211 (83e:80002) 
[C-E-L] M. G. Crandall, L. C. Evans and P. L. Lions, Some properties of viscosity solutions of Hamilton-Jacobi equations, Trans. AMS 282 (1984), 487-502. MR 0732102 (86a:35031)

[C-L] M. G. Crandall and P. L. Lions, Viscosity solutions of Hamilton-Jacobi equations, Trans. AMS 277 (1983), 1-42. MR 0690039 (85g:35029)

[D] W. Day, Entropy and Partial Differential Equations, Pitman Research Notes in Mathematics Series 295, Longman, 1993. MR 1413296 (97h:35179)

[D-S] W. Day and M. Šilhavý, Efficiency and existence of entropy in classical thermodynamics, Arch. Rat. Mech. Analysis 66 (1977), 73-81. MR 0449196 (56:7501)

[D-Z] A. Dembo and O. Zeitouni, Large Deviation Techniques and Applications, Jones and Barlett Publishers, 1993. MR 1202429 (95a:60034)

[D-H-M-T] M. DiBattista, K. Haven, A. Majda and B. Turkington, Statistical equilibrium predictions of jets and spots on Jupiter, Proc. Nat. Acad. Sciences 98 (2001), 12346-12350. MR 1858819 (2002h:85004)

[D-Ze] R. Dittman and M. Zemansky, Heat and Thermodynamics, McGraw-Hill, 1997.

[Ec] K. Ecker, Regularity Theory for Mean Curvature Flow, Birkhauser, 2004. MR 2024995

[El] R. S. Ellis, Entropy, Large Deviations and Statistical Mechanics, Springer, 1985. MR 0793553 (87d:82008)

[Er] J. L. Ericksen, Introduction to the Thermodynamics of Solids, Revised edition, Springer, 1998. MR 1607500 (98k:73009)

[E1] L. C. Evans, Partial Differential Equations, AMS Press, 1998. MR 1625845 (99e:35001)

[E2] L. C. Evans, The perturbed test function method for viscosity solutions of nonlinear PDE, Proc. Royal Soc. Edinburgh 111 (1989), 359-375. MR 1007533 (91c:35017)

[E-P] L. C. Evans and M. Portilheiro, Irreversibility and hysteresis for a forward-backward diffusion PDE, to appear.

[F-L] M. Feinberg and R. Lavine, Thermodynamics based on the Hahn-Banach Theorem: the Clausius inequality, Arch. Rat. Mech. Analysis 82 (1983), 203-293. MR 0690294 (84f:80001)

[F] M. I. Freidlin, Probabilistic approach to the small viscosity asymptotics for NavierStokes equations, Nonlinear Analysis 30 (1997), 4069-4076. MR 1603550 (99g:76033)

[F-W] M. I. Freidlin and A. D. Wentzell, Random Perturbations of Dynamical Systems, Springer, 1984. MR 0722136 (85a:60064)

[Gu] M. Gurtin, Thermodynamics of Evolving Phase Boundaries in the Plane, Oxford, 1993. MR 1402243 (97k:73001)

[G-W] M. Gurtin and W. Williams, An axiomatic foundation for continuum thermodynamics, Arch. Rat. Mech. Analysis 26 (1967), 83-117. MR 0214335 (35:5186)

[Lx1] P. Lax, Shock waves and entropy, in Contributions to Nonlinear Functional Analysis, Academic Press (1971), 603-634. MR 0393870 (52:14677)

[Lx2] P. Lax, Shock waves, increase of entropy and loss of information, in Seminar on Nonlinear PDE, ed. by S. S. Chern, MSRI Publications, Springer (1984), 129-171. MR 0765233 (86b:35130)

[L-Y] E. Leib and J. Yngvason, A guide to entropy and the second law of thermodynamics, Notices AMS 45 (1998), 571-581. MR 1616141 (99c:80002)

[L-Ya] P. Li and S. T. Yau, On the parabolic kernel of the Schrödinger operator, Acta. Math. 156 (1986), 153-201. MR 0834612 (87f:58156)

[Li] F. Lin, A new proof of the Caffarelli-Kohn-Nirenberg Theorem, Comm. Pure and Applied Math. 51 (1998), 241-257. MR 1488514 (98k:35151)

[L] P. L. Lions, On Euler Equations and Statistical Physics, Cattedra Galileiana, Pisa (1998). MR 1657480 (99m:35189)

[L-P-S] P. L. Lions, B. Perthame and P. E. Souganidis, Existence and stability of entropy solutions for the hyperbolic systems of isentropic gas dynamics in Eulerian and Lagrandian coordinates, Comm. Pure Appl. Math. 49 (1996), 599-638. MR 1383202 (97e:35107)

[L-P-T1] P. L. Lions, B. Perthame and E. Tadmor, A kinetic formulation of multidimensional conservation laws and related equations, Journal AMS 7 (1994), 169-191. MR 1201239 (94d:35100) 
[L-P-T2] P. L. Lions, B. Perthame and E. Tadmor, Kinetic formulation of isentropic gas dynamics and p-systems, Comm. Math. Physics 163 (1994), 415-431. MR 1284790 (95c:76093)

[Li-Y] T-P. Liu and T. Yang, A new entropy functional for a scalar conservation law, Comm. Pure and Appl. Math. 52 (1999), 1427-1442. MR 1702712 (2000e:35139)

[M-P] C. Marchioro and M. Pulvirenti, Mathematical Theory of Incompressible Nonviscous Fluids, Springer, 1994. MR 1245492 (94k:76001)

[M-R] A. Mikelic and R. Robert, On the equations describing a relaxation toward a statistical equilibrium state in two-dimensional perfect fluid dynamics, SIAM J. Math. Analysis 29 (1998), 1238-1255. MR 1628271 (99d:76051)

[NC-P] A. Novick Cohen and R. Pego, Stable patterns in a viscous diffusion equation, Transactions AMS 324 (1991), 331-351. MR 1015926 (91f:35152)

[O-S] S. Osher and J. Sethian, Fronts propagating with curvature-dependent speed: algorithms based on Hamilton-Jacobi formulations, Comput. Phys. 79 (1988) 12-49. MR 0965860 (89h:80012)

[O] D. Owen, A First Course in the Mathematical Foundations of Thermodynamics, Springer, 1984. MR 0725673 (85m:80001)

[P] B. Perthame, Kinetic Formulation of Conservation Laws, Oxford University Press, 2002.

[P-T $] \quad$ B. Perthame and E. Tadmor, A kinetic equation with kinetic entropy functions for scalar conservation laws, Comm. Math. Physics 136 (1991), 501-517. MR 1099693 (92d:82095)

[Pe] M. A. Peterson, Analogy between thermodynamics and mechanics, American J. Physics 47 (1979), 488-490. MR 0532721 (80b:80014)

[Pi] M. A. Pinsky, Lectures on Random Evolution, World Scientific, 1992. MR 1143780 (93b:60160)

[Pl] P. I. Plotnikov, Passing to the limit with respect to the viscosity in an equation with variable parabolicity direction, Differential Equations 30 (1994), 614-622.

[S] V. Scheffer, Hausdorff measure and the Navier-Stokes equations, Comm. Math. Phys. 55 (1977), 97-112. MR 0510154 (58:23176)

[S1] J. Serrin, Foundations of Classical Thermodynamics, Lecture Notes, Math. Department, U. of Chicago, 1975. MR 0672251 (58:32431)

[S2] J. Serrin, Conceptual analysis of the classical second laws of thermodynamics, Arch. Rat. Mech. Analysis 70 (1979), 353-371. MR 0574162 (81g:80002)

$[\mathrm{Tu}] \quad$ B. Turkington, Statistical equilibrium and coherent states in two-dimensional turbulence, Comm. in Pure and Applied Math. 52 (1999), 781-809. MR 1682816 (99k:76082)

[V] S. R. S. Varadhan, Large Deviations and Applications, SIAM, 1984. MR 0758258 (86h:60067b)

[Vi] C. Villani, Topics in Optimal Transportation, AMS Press, 2003. MR 1964483 (2004e:90003)

[Vs] A. Visintin, Differential Models of Hysteresis, Springer, 1994. MR 1329094 (96h:47001)

[W] A. Wightman, Convexity and the notion of equilibrium state in thermodynamics and statistical mechanics, Introduction to R. B. Israel, Convexity in the Theory of Lattice Gases, Princeton U. Press, 1979. MR 0517873 (80i:82002)

Department of Mathematics, University of California, Berkeley, Berkeley, CaliFORNIA 94720-0001 